\title{
NLO gravitational quartic-in-spin interaction
}

\author{
Michèle Levi $^{a}$ and Fei Teng ${ }^{b}$ \\ ${ }^{a}$ Niels Bohr International Academy, Niels Bohr Institute, University of Copenhagen, \\ Blegdamsvej 17, 2100 Copenhagen, Denmark \\ ${ }^{b}$ Department of Physics and Astronomy, Uppsala University, \\ 75108 Uppsala, Sweden \\ E-mail: michelelevi@nbi.ku.dk, fei.teng@physics.uu.se
}

\begin{abstract}
In this paper we derive for the first time the complete gravitational quarticin-spin interaction of generic compact binaries at the next-to-leading order in the postNewtonian (PN) expansion. The derivation builds on the effective field theory for gravitating spinning objects, and its recent extensions, in which new type of worldline couplings should be considered, as well as on the extension of the effective action of a spinning particle to quadratic order in the curvature. The latter extension entails a new Wilson coefficient that appears in this sector. This work pushes the precision frontier with spins at the fifth PN (5PN) order for maximally-spinning compact objects, and at the same time informs us of the gravitational Compton scattering with higher spins.
\end{abstract}

KEYwords: Classical Theories of Gravity, Effective Field Theories, Black Holes, Scattering Amplitudes

ArXiv EPrint: 2008.12280 


\section{Contents}

1 Introduction 1

2 The EFT of gravitating spinning objects 3

2.1 Extending the EFT of a spinning particle 5

3 Elementary worldline couplings $\quad 7$

$\begin{array}{ll}3.1 & \text { One-graviton exchange } \\ 3.2 & 7 \text {-gaviton }\end{array}$

$\begin{array}{lll}3.2 & \text { Two-graviton exchange } & 14\end{array}$

$\begin{array}{lll}3.3 & \text { Cubic self-interaction } & 15\end{array}$

4 Composite worldline couplings $\quad 16$

5 Quadratic-in-curvature worldline couplings $\quad 19$

6 Next-to-leading gravitational quartic-in-spin action 20

$\begin{array}{lll}7 & \text { Conclusions } & 24\end{array}$

\section{Introduction}

In this work we pursue two interwoven objectives, each of which has a different nature. The first objective falls under the timely phenomenological efforts to improve the theoretical precision for modeling gravitational waveforms, that are currently successfully being used to measure gravitational waves (GWs) from mergers of compact binaries as of 2016 [1]. The post-Newtonian (PN) approximation of classical Gravity [2] via the effective-one body approach [3] provides the crucial input for the generation of GW templates, and accordingly significant progress has been carried out recently in pushing the state of the art, with the current precision frontier at the fourth and a half PN (4.5PN) [4-6] and the 5PN [7-12] orders in the accuracy of the orbital dynamics. The complete state of the art of the generic orbital dynamics of a compact binary to date is captured in table 1. In order to complete a certain PN accuracy, the sectors that are shown in table 1 should be addressed across its diagonals, which stands for two orthogonal types of challenges: the challenge of going along the horizontal axis of the table is that of computational multi-loop technology, whereas the challenge of going along the vertical axis of the table involves improving the conceptual understanding of spin in gravity and higher-spin theory.

This brings us to the second objective of the present work, which is the theoretical effort to understand what happens in gravitational interactions when higher spins are involved. Within classical gravity the first work to provide the LO quadratic-in-spin interaction, including the spin-induced quadrupole was carried out decades ago [16]. This was considered 


\begin{tabular}{|l|r|r|r|r|r|}
\hline \multicolumn{1}{|c|}{$\boldsymbol{l}$} & $\left(\mathrm{N}^{\mathbf{0}}\right) \mathrm{LO}$ & $\mathrm{N}^{(1)} \mathrm{LO}$ & $\mathrm{N}^{2} \mathrm{LO}$ & $\mathrm{N}^{3} \mathrm{LO}$ & $\mathrm{N}^{4} \mathrm{LO}$ \\
\hline $\mathrm{S}^{\mathbf{0}}$ & 1 & 0 & 3 & 0 & 25 \\
\hline $\mathrm{S}^{\mathbf{1}}$ & 2 & 7 & 32 & $\mathbf{1 7 4}$ & \\
\hline $\mathrm{S}^{\mathbf{2}}$ & 2 & 2 & $\mathbf{1 8}$ & $\mathbf{5 2}$ & \\
\hline $\mathrm{S}^{\mathbf{3}}$ & 4 & $\mathbf{2 4}$ & & & \\
\hline $\mathrm{S}^{\mathbf{4}}$ & 3 & $\mathbf{5}$ & & & \\
\hline
\end{tabular}

Table 1. The complete state of the art of PN orbital dynamics of generic compact binaries. The PN corrections enter at the order $n+l+\operatorname{Parity}(l) / 2$, with the parity 0 or 1 for even or odd $l$, respectively. The higher-order sectors with the entries in boldface have been uniquely addressed in $[4,5,10,13]$ and in the present work via the EFT of gravitating spinning objects [14]. The entries in the table indicate the loop computational scale within this framework, namely the number of highest-loop graphs in each sector. The sectors up to the current complete state of the art at the $4 \mathrm{PN}$ order, except the top right one, are available in the public EFTofPNG code at https: //github.com/miche-levi/pncbc-eftofpng [15].

much later within the effective field theory (EFT) approach to PN Gravity [17], whose extension to the spinning case was first approached in [18], with effective parameters referred to as Wilson coefficients, similar to the spin-induced quadrupolar deformation parameter in [19]. The EFT of gravitating spinning objects was then introduced in [14], where the tower of gravitational couplings to all orders in spin, that are linear in the curvature, was provided. Later, a new spinor-helicity formalism for massive particles of any spin was introduced in [20], and was instrumental to recent studies within the language of scattering amplitudes of classical spin effects [21-27]. Scattering amplitudes involving a quantum spin of $s=l / 2$ correspond to classical interactions with spin to the $l$ th order, and hence the gray area in table 1 corresponds to where the gravitational Compton scattering (see figure 1) with a quantum spin $s>1$ is required, as of the one-loop level. The fundamental issue is that the gravitational Compton amplitude cannot be uniquely fixed for $s>2$, related with the tension in formulating a perturbative UV completion of gravity with higher spins [20].

Interestingly, at the classical level the gray area in table 1 also corresponds to where the linear momentum can no longer be taken as independent of the spin, and new types of complicate contributions arise in the interactions, as already pointed out in [14]. At this stage it is not clear whether one gets a unique well-defined classical result, which resonates with the difficulty of uniquely fixing the graviton Compton amplitude with higher spins, and thus these two inquiries may inform each other.

This work directly builds on the EFT of gravitating spinning objects introduced in [14], its implementation on [28] at LO, and the recent extension to the cubic-in-spin interaction at NLO in [4], as well as on the recent study of the NNNLO quadratic-in-spin interaction in [10], to get the quartic-in-spin interaction at the NLO. This sector enters at the 5PN order for maximally-spinning compact objects, beyond the current complete PN state of the art in general, and with spins in particular [29]. This paper, that pushes PN precision with higher spin, extends the body of work of various past studies in [4, 10, 13, 14, 28, 30-37], 


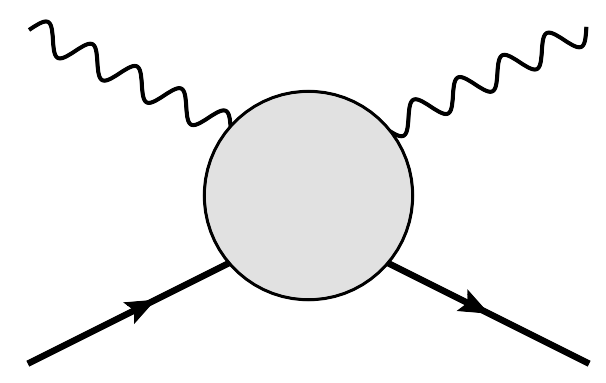

Figure 1. The gravitational Compton scattering with two massive spin particles and two gravitons.

where here we derive the complete quartic-in-spin sector, including all interactions with all possible spin-induced higher-order multipoles up to and including the hexadecapole. This sector is also the next complicate one after that in [4] in the intriguing gray area of table 1 , and thus beyond pushing the state of the art in PN theory, we should be alert to the new conceptual features that show up in it.

The paper is organized as follows. In section 2 we review briefly the formulation of the EFT of spinning gravitating objects from [14], and outline the ingredients relevant to this sector. Notably, in section 2.1 we extend the effective theory of a spinning particle, in order to extract new contributions to the current sector. In section 3 we present the full perturbative expansion with the elementary worldline couplings of the EFT, where the linear momentum is taken as independent of the spin, and in section 4 we handle new contributions due to composite worldline couplings that emerge, similar to what was first seen in the NLO cubic-in-spin sector [4]. In section 5 we consider further new contributions arising from new worldline couplings that are quadratic in the curvature. The final result for the total effective action of the complete NLO gravitational quartic-in-spin sector is provided in section 6 , and we conclude the work in section 7. An ancillary file of Mathematica notebook that contains all the results of this paper is included in the arXiv submission.

\section{The EFT of gravitating spinning objects}

The evaluation of this sector, that contains spins at quartic order with leading gravitational nonlinearities, builds on the EFT of gravitating spinning objects formulated in [14], its implementation from LO to NNLO up to the complete state of the art at the 4PN order accomplished in $[13,14,38]$, the LO complete cubic- and quartic-in-spin sectors obtained in [28], as well as on the recent complete NLO cubic-in-spin sector obtained in [4] at the 4.5PN order, and the recent studies of the NNNLO spin-orbit and quadratic-in-spin sectors in $[5,10]$ at 4.5 and $5 \mathrm{PN}$ orders. We follow all the conventions and gauge choices in the aforementioned papers, and we also use the beneficial Kaluza-Klein (KK) decomposition of the field to scalar, vector and tensor components, as in all high-order PN computations in the EFT approach [39-41].

We start from a two-particle effective action [41], in which each of the objects is captured by the one-particle effective action of a spinning particle, that was derived in [14]. 
This effective action contains a pure gravitational piece, and two copies of a point-particle action that captures the coupling of gravity to the worldline degrees of freedom (DOFs). The Feynman rules for the propagators and self-interaction vertices derived from the purely gravitational action are found in [42] and [38]. The effective action of a spinning particle, including its spin-induced non-minimal coupling, and in which the gauge freedom of the rotational DOFs was introduced into the action, has the form [14]

$$
S_{\mathrm{pp}}(\sigma)=\int d \sigma\left[-m \sqrt{u^{2}}-\frac{1}{2} \hat{S}_{\mu \nu} \hat{\Omega}^{\mu \nu}-\frac{\hat{S}^{\mu \nu} p_{\nu}}{p^{2}} \frac{D p_{\mu}}{D \sigma}+L_{\mathrm{SI}}\right]
$$

with the four-velocity $u^{\mu}$, the conjugate linear momentum $p_{\mu}$ and the generic rotational DOFs, denoted with a hat, e.g. $\hat{S}_{\mu \nu}$. The spin-induced part, labeled by "SI", contributes to this sector via its three leading terms [14]:

$$
L_{\mathrm{SI}}=-\frac{C_{\mathrm{ES}^{2}}}{2 m} \frac{E_{\mu \nu}}{\sqrt{u^{2}}} S^{\mu} S^{\nu}-\frac{C_{B S^{3}}}{6 m^{2}} \frac{D_{\lambda} B_{\mu \nu}}{\sqrt{u^{2}}} S^{\mu} S^{\nu} S^{\lambda}+\frac{C_{\mathrm{ES}^{4}}}{24 m^{3}} \frac{D_{\kappa} D_{\lambda} E_{\mu \nu}}{\sqrt{u^{2}}} S^{\mu} S^{\nu} S^{\lambda} S^{\kappa},
$$

in which we use the electric and magnetic components of the curvature tensor, with the Levi-Civita tensor density in curved spacetime in the latter, for definite parity

$$
\begin{aligned}
E_{\mu \nu} & \equiv R_{\mu \alpha \nu \beta} u^{\alpha} u^{\beta}, \\
B_{\mu \nu} & \equiv \frac{1}{2} \epsilon_{\alpha \beta \gamma \mu} R_{\delta \nu}^{\alpha \beta} u^{\gamma} u^{\delta},
\end{aligned}
$$

of even and odd parity, respectively, and a classical version of the Pauli-Lubanski pseudovector, $S^{\mu}$, as defined first in [28]:

$$
S_{\mu}=\frac{1}{2} \epsilon_{\alpha \beta \gamma \mu} S^{\alpha \beta} \frac{p^{\gamma}}{p} .
$$

Note that this definition is with a reverse sign with respect to the one detailed in [14], which was implemented there up to the quadratic-in-spin order, where the sign choice does not make a difference. Further, note that $S^{\alpha \beta}$ here is not the generic spin variable, but rather its projection to the rest frame, see eq. (3.29) in [14]

We remind the extra term with a covariant derivative of the linear momentum in eq. (2.1), which is essentially the Thomas precession, that was derived from the introduction of gauge freedom to the rotational DOFs in [14] (later recovered in e.g. [24] as "Hilbert space matching"). This term is relevant to all orders in spin, including all finite size spin effects, though it does not encapsulate any UV physics, but rather accounts for the extended measure of a relativistic gravitating spinning object.

Since we compute here the complete NLO quartic-in-spin sector our graphs contain all spin-induced higher-multipoles up to the hexadecapole, in addition to the mass and spin. Therefore we need the Feynman rules of worldline-graviton coupling to NLO for all of these multipoles, including new rules for the hexadecapole couplings. The Feynman rules for the mass, the spin and the spin-induced quadrupole are found e.g. in [14, 38, 43], and [13]. The spin-induced octupole couplings are found in $[4,28]$. In this work the Feynman rule of the 
scalar component of the KK fields, which appeared at LO in [28], should be extended to the next PN order and is given as follows:

$$
\begin{array}{r}
1-\int t-\frac{C_{\mathrm{ES}}}{24 m^{3}}\left[S_{i} S_{j} S_{k} S_{l}\left(\partial_{i} \partial_{j} \partial_{k} \partial_{l} \phi\left(1+\frac{3}{2} v^{2}\right)-2 v^{i} v^{m} \partial_{m} \partial_{j} \partial_{k} \partial_{l} \phi\right)\right. \\
\left.+S^{2} S_{j} S_{k}\left(v^{m} v^{l} \partial_{m} \partial_{l} \partial_{j} \partial_{k} \phi+2 v^{l} \partial_{l} \partial_{j} \partial_{k} \partial_{t} \phi+\partial_{j} \partial_{k} \partial_{t}^{2} \phi\right)\right],
\end{array}
$$

where the crossed black box represents the spin-induced hexadecapole. In this Feynman rule and all the rules that follow in this paper, all indices are Euclidean, the spin is fixed in the canonical gauge in the local frame, and $S_{i} \equiv \epsilon_{i j k} S_{j k}$ (for more details on these points, see [14]). We also need new rules for the one-graviton coupling of the KK vector field, and for the two-graviton coupling with the KK scalar field, which are given as follows:

$$
\begin{gathered}
=\int d t \frac{C_{\mathrm{ES}^{4}}}{24 m^{3}} S_{i} S_{j} S_{k} S_{l}\left[v^{m} \partial_{i} \partial_{j} \partial_{k} \partial_{l} A_{m}-v^{m} \partial_{m} \partial_{j} \partial_{k} \partial_{l} A_{i}-\partial_{j} \partial_{k} \partial_{l} \partial_{t} A_{i}\right], \\
=\int d t-\frac{C_{\mathrm{ES}^{4}}}{24 m^{3}}\left[S_{i} S_{j} S_{k} S_{l}\left(16 \partial_{i} \partial_{j} \partial_{k} \phi \partial_{l} \phi+10 \partial_{i} \partial_{j} \phi \partial_{k} \partial_{l} \phi+5 \phi \partial_{i} \partial_{j} \partial_{k} \partial_{l} \phi\right)\right. \\
\left.-S^{2} S_{j} S_{k}\left(7 \partial_{j} \partial_{k} \partial_{n} \phi \partial_{n} \phi+4 \partial_{j} \partial_{n} \phi \partial_{k} \partial_{n} \phi\right)\right] .
\end{gathered}
$$

Note the dominant role that the KK scalar field plays in the coupling to the evenparity hexadecapole, similar to the couplings to the even-parity mass monopole, and spininduced quadrupole.

For this sector we also need to extend the non-minimal coupling part of the spinning particle action, and add higher-dimensional operators beyond the linear-in-Riemann ones, which were derived to all orders in spin in [14], but we also need to address the new feature that became relevant as of the NLO cubic-in-spin sector (the simplest corner of the gray area in table 1) [4]. For the latter we need to take into account corrections to the linear momentum that are linear in Riemann and higher-order in the spin similar to [4], which was already explicitly noted in [14]. These corrections give rise to what we will refer to as "composite" worldline couplings, which are considered in section 4, after the "elementary" worldline couplings, in which the linear momentum is still independent of the spin (the white area of table 1), are covered in section 3.

\subsection{Extending the EFT of a spinning particle}

As noted above at the 5PN order the effective action, or more specifically, the non-minimal coupling part of the effective action of a spinning particle, that is given in eq. (2.2), should be extended as done in [10]. This extension should include operators that are higher-order in the curvature components, and describe the tidal deformations of the compact object. This extension should only include operators that are quadratic in the curvature, since here only corrections up to order $G^{2}$ are considered. Following the symmetries and the 
logic detailed in [14], and implemented in the recent extension in [10], we find that the new terms to quartic order in spin are of the form:

$$
\begin{aligned}
& L_{\mathrm{NMC}\left(\mathrm{R}^{2}\right)}=C_{E^{2}} \frac{E_{\alpha \beta} E^{\alpha \beta}}{\sqrt{u^{2}}}+C_{B^{2}} \frac{B_{\alpha \beta} B^{\alpha \beta}}{{\sqrt{u^{2}}}^{3}}+\ldots \\
& +C_{E^{2} S^{2}} S^{\mu} S^{\nu} \frac{E_{\mu \alpha} E_{\nu}^{\alpha}}{{\sqrt{u^{2}}}^{3}}+C_{B^{2} S^{2}} S^{\mu} S^{\nu} \frac{B_{\mu \alpha} B_{\nu}^{\alpha}}{{\sqrt{u^{2}}}^{3}} \\
& +C_{E^{2} S^{4}} S^{\mu} S^{\nu} S^{\kappa} S^{\rho} \frac{E_{\mu \nu} E_{\kappa \rho}}{{\sqrt{u^{2}}}^{3}}+C_{B^{2} S^{4}} S^{\mu} S^{\nu} S^{\kappa} S^{\rho} \frac{B_{\mu \nu} B_{\kappa \rho}}{{\sqrt{u^{2}}}^{3}} \\
& +C_{\nabla E B S} S^{\mu} \frac{D_{\mu} E_{\alpha \beta} B^{\alpha \beta}}{{\sqrt{u^{2}}}^{3}}+C_{E \nabla B S} S^{\mu} \frac{E_{\alpha \beta} D_{\mu} B^{\alpha \beta}}{{\sqrt{u^{2}}}^{3}} \\
& +C_{\nabla E B S^{3}} S^{\mu} S^{\nu} S^{\kappa} \frac{D_{\kappa} E_{\mu \alpha} B_{\nu}^{\alpha}}{{\sqrt{u^{2}}}^{3}}+C_{E \nabla B S^{3}} S^{\mu} S^{\nu} S^{\kappa} \frac{E_{\mu \alpha} D_{\kappa} B_{\nu}^{\alpha}}{{\sqrt{u^{2}}}^{3}} \\
& +C_{(\nabla E)^{2} S^{2}} S^{\mu} S^{\nu} \frac{D_{\mu} E_{\alpha \beta} D_{\nu} E^{\alpha \beta}}{{\sqrt{u^{2}}}^{3}}+C_{(\nabla B)^{2} S^{2}} S^{\mu} S^{\nu} \frac{D_{\mu} B_{\alpha \beta} D_{\nu} B^{\alpha \beta}}{{\sqrt{u^{2}}}^{3}} \\
& +C_{(\nabla E)^{2} S^{4}} S^{\mu} S^{\nu} S^{\kappa} S^{\rho} \frac{D_{\kappa} E_{\mu \alpha} D_{\rho} E_{\nu}^{\alpha}}{{\sqrt{u^{2}}}^{3}} \\
& +C_{(\nabla B)^{2} S^{4}} S^{\mu} S^{\nu} S^{\kappa} S^{\rho} \frac{D_{\kappa} B_{\mu \alpha} D_{\rho} B_{\nu}^{\alpha}}{{\sqrt{u^{2}}}^{3}}+\ldots
\end{aligned}
$$

with new tidal Wilson coefficients involved with these new operators, which in contrast to those in eq. (2.2) - are currently defined to absorb all numerical and mass factors.

As in [10] in the first line of eq. (2.9) the leading mass-induced quadrupolar tidal deformations, which are known to enter at the 5PN order are written [41], suppressing higher-order mass-induced tidal operators, that were provided in [44]. We have then written the adiabatic tidal operators with spin up to the quartic order. From power-counting considerations, which were detailed in $[14,41]$, it can be deduced that the second and third lines of eq. (2.9) contribute as of the 5PN order, whereas the fourth and fifth lines, and the sixth and seventh lines, of eq. (2.9), contribute as of the 6.5PN and 7PN orders, respectively. Therefore at the 5PN order considered in this work there are two new operators which are quartic in the spin and quadratic in the curvature that are given by

$$
L_{\mathrm{S}^{4}\left(\mathrm{R}^{2}\right)}=\frac{C_{E^{2} S^{4}}}{24 m^{3}} S^{\mu} S^{\nu} S^{\kappa} S^{\rho} \frac{E_{\mu \nu} E_{\kappa \rho}}{{\sqrt{u^{2}}}^{3}}+\frac{C_{B^{2} S^{4}}}{24 m^{3}} S^{\mu} S^{\nu} S^{\kappa} S^{\rho} \frac{B_{\mu \nu} B_{\kappa \rho}}{{\sqrt{u^{2}}}^{3}},
$$

in which the numerical and mass factors have now been adjusted to render the Wilson coefficients dimensionless.

The consequent new Feynman rule that contributes from eq. (2.10) is then a twograviton coupling of the KK scalar field given by

$$
=\int d t \frac{C_{\mathrm{E}^{2} \mathrm{~S}^{4}}}{24 m^{3}}\left[S_{i} S_{j} S_{k} S_{l} \phi_{, i j} \phi_{, k l}-2 S^{2} S_{i} S_{j} \phi_{, i j} \phi_{, k k}+S^{4} \phi_{, i i} \phi_{, j j}\right] \text {, }
$$


that arises from the quadratic electric operator, and is represented here by the black "star of Lakshmi". As to the quadratic magnetic operator in eq. (2.10), it contributes a twograviton coupling of the KK vector field, which will become relevant only at the $6 \mathrm{PN}$ order, namely beyond the present sector.

\section{Elementary worldline couplings}

Let us first evaluate the Feynman graphs from the diagrammatic expansion of the effective action, that contain the elementary worldline couplings, i.e. those obtained under the leading approximation of the linear momentum that is independent of spin. With spins all of the three relevant topologies up to the $G^{2}$ order are realized even with the useful KK decomposition [14, 41, 43, 45], and we have here a total of $23=10+8+5$ graphs, distributed among the topologies of one- and two-graviton exchanges, and cubic self-interaction, as shown in figures 2-4 (drawn with Jaxodraw [46, 47] based on [48]), respectively. As noted in table 1 only 5 of the graphs require a one-loop evaluation, though as we consider the nonlinear graphs in the sector, the options to assemble a quartic-in-spin interaction multiply.

At the linear level there are only three types of interaction, similar to the LO in [28], namely either a quadrupole-quadrupole, an octupole-dipole or a hexadecapole-monopole interaction. These graphs are easily constructed following the nice analogies among these types of interaction according to the parity of the multipole moments involved [4, 28]. Yet, more types of interaction emerge once we proceed to the nonlinear level, in which there are interactions with various multipoles on two different points of the worldline, which occurs as of the NLO spin-squared sector $[4,13,14]$, and which add up to an interaction that is quartic in the spin, such as a spin and a spin-induced quadrupole, or two spin-induced quadrupoles on the same worldline.

Note that all the graphs in this sector are to be included together with their mirror images, in which the worldline labels are exchanged, $1 \leftrightarrow 2$, and that more details on the generation and the evaluation of the Feynman graphs can be found in [41] and references therein.

\subsection{One-graviton exchange}

There are 10 graphs of one-graviton exchange in this sector, as seen in figure 2, in which most already contain time derivatives to be evaluated. As in previous works of one of the authors, all of the higher-order time derivative terms that appear in the evaluations of the graphs are kept, to be treated rigorously later via the procedure of redefinitions of variables, that was explained in detail in [49]. Notice that there are 3 graphs that appeared at the LO, to which we add the quadratic time insertions on the propagators at graphs 2(a2), (b2), (c2), and a new hexadecapole coupling of the KK vector field at graph 2(c3).

The graphs in figure 2 have the following values:

$$
\begin{aligned}
\text { Fig. } 2(\mathrm{a} 1)= & \frac{3 C_{1\left(\mathrm{ES}^{2}\right)} C_{2\left(\mathrm{ES}^{2}\right)}}{8} \frac{G}{m_{1} m_{2} r^{5}}\left[70\left(\vec{S}_{1} \cdot \vec{n}\right)^{2}\left(\vec{S}_{2} \cdot \vec{n}\right)^{2}\left(1+\frac{3 v_{1}^{2}}{2}+\frac{3 v_{2}^{2}}{2}\right)\right. \\
& -10\left(\vec{S}_{1} \cdot \vec{n}\right)^{2} S_{2}^{2}\left(1+\frac{3 v_{1}^{2}}{2}+\frac{5 v_{2}^{2}}{2}-7\left(\vec{v}_{2} \cdot \vec{n}\right)^{2}\right)
\end{aligned}
$$




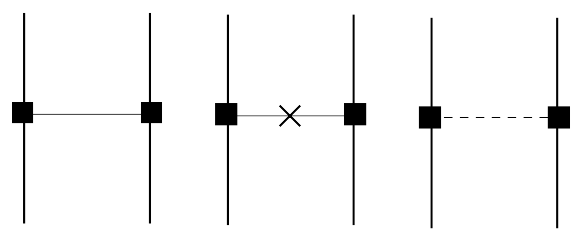

(a1)

(a2)

(a3)

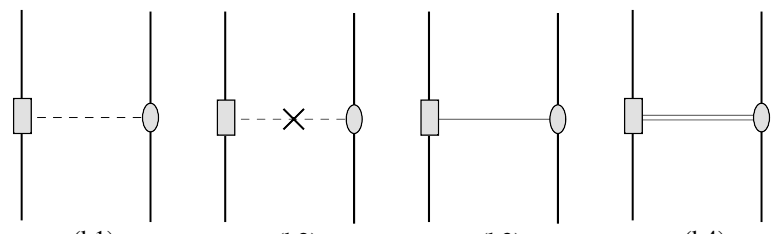

(b1)

(b2)

(b3)

(b4)

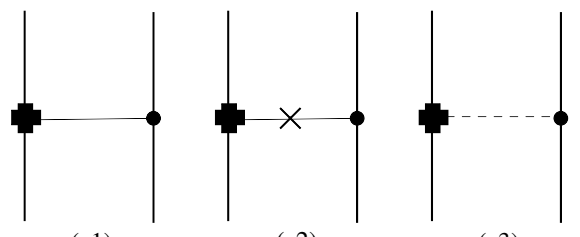

(c1)

(c2)

(c3)

Figure 2. The Feynman graphs of one-graviton exchange, that contribute to the NLO quartic-inspin interaction at the $5 \mathrm{PN}$ order for maximally-rotating compact objects. At the linear level we only have three types of interaction, similar to the LO in [28], of either a quadrupole-quadrupole, octupole-dipole or a hexadecapole-monopole type. The graphs are easily constructed following the nice analogies pointed out in [28] among the interactions according to the parity of the multipole moments involved. Notice that we have here the three graphs that appeared at the LO with the quadratic time insertions on the propagators at graphs (a2), (b2), (c2), and a new hexadecapole coupling with the KK vector field at graph (c3).

$$
\begin{aligned}
& -40\left(\vec{S}_{1} \cdot \vec{n}\right)\left(\vec{S}_{1} \cdot \vec{S}_{2}\right)\left(\vec{S}_{2} \cdot \vec{n}\right)\left(1+\frac{3 v_{1}^{2}}{2}+\frac{3 v_{2}^{2}}{2}\right) \\
& -70\left(\vec{S}_{1} \cdot \vec{n}\right)^{2}\left(\vec{S}_{2} \cdot \vec{n}\right)\left(\vec{S}_{2} \cdot \vec{v}_{2}\right)\left(\vec{v}_{2} \cdot \vec{n}\right)+10\left(\vec{S}_{1} \cdot \vec{n}\right)^{2}\left(\vec{S}_{2} \cdot \vec{v}_{2}\right)^{2} \\
& +10\left(\vec{S}_{1} \cdot \vec{n}\right)\left(\vec{S}_{1} \cdot \vec{v}_{1}\right) S_{2}^{2}\left(\vec{v}_{1} \cdot \vec{n}\right)-40\left(\vec{S}_{1} \cdot \vec{n}\right)\left(\vec{S}_{1} \cdot \vec{v}_{2}\right) S_{2}^{2}\left(\vec{v}_{2} \cdot \vec{n}\right) \\
& -70\left(\vec{S}_{1} \cdot \vec{n}\right)\left(\vec{S}_{1} \cdot \vec{v}_{1}\right)\left(\vec{S}_{2} \cdot \vec{n}\right)^{2}\left(\vec{v}_{1} \cdot \vec{n}\right)+20\left(\vec{S}_{1} \cdot \vec{n}\right)\left(\vec{S}_{1} \cdot \vec{v}_{1}\right)\left(\vec{S}_{2} \cdot \vec{n}\right)\left(\vec{S}_{2} \cdot \vec{v}_{1}\right) \\
& +20\left(\vec{S}_{1} \cdot \vec{n}\right)\left(\vec{S}_{1} \cdot \vec{v}_{2}\right)\left(\vec{S}_{2} \cdot \vec{n}\right)\left(\vec{S}_{2} \cdot \vec{v}_{2}\right)+20\left(\vec{S}_{1} \cdot \vec{n}\right)\left(\vec{S}_{1} \cdot \vec{S}_{2}\right)\left(\vec{S}_{2} \cdot \vec{v}_{2}\right)\left(\vec{v}_{2} \cdot \vec{n}\right) \\
& +10\left(\vec{S}_{1} \cdot \vec{v}_{1}\right)^{2}\left(\vec{S}_{2} \cdot \vec{n}\right)^{2}-2\left(\vec{S}_{1} \cdot \vec{v}_{1}\right)^{2} S_{2}^{2}+20\left(\vec{S}_{1} \cdot \vec{v}_{1}\right)\left(\vec{S}_{1} \cdot \vec{S}_{2}\right)\left(\vec{S}_{2} \cdot \vec{n}\right)\left(\vec{v}_{1} \cdot \vec{n}\right) \\
& -4\left(\vec{S}_{1} \cdot \vec{v}_{1}\right)\left(\vec{S}_{1} \cdot \vec{S}_{2}\right)\left(\vec{S}_{2} \cdot \vec{v}_{1}\right)-4\left(\vec{S}_{1} \cdot \vec{v}_{2}\right)\left(\vec{S}_{1} \cdot \vec{S}_{2}\right)\left(\vec{S}_{2} \cdot \vec{v}_{2}\right) \\
& +4\left(\vec{S}_{1} \cdot \vec{v}_{2}\right)^{2} S_{2}^{2}+4\left(\vec{S}_{1} \cdot \vec{S}_{2}\right)^{2}\left(1+\frac{3 v_{1}^{2}}{2}+\frac{3 v_{2}^{2}}{2}\right) \\
& -10 S_{1}^{2}\left(\vec{S}_{2} \cdot \vec{n}\right)^{2}\left(1+\frac{5 v_{1}^{2}}{2}+\frac{3 v_{2}^{2}}{2}-7\left(\vec{v}_{1} \cdot \vec{n}\right)^{2}\right)+4 S_{1}^{2}\left(\vec{S}_{2} \cdot \vec{v}_{1}\right)^{2}-2 S_{1}^{2}\left(\vec{S}_{2} \cdot \vec{v}_{2}\right)^{2} \\
& -40 S_{1}^{2}\left(\vec{S}_{2} \cdot \vec{n}\right)\left(\vec{v}_{1} \cdot \vec{n}\right)\left(\vec{S}_{2} \cdot \vec{v}_{1}\right)+10 S_{1}^{2}\left(\vec{S}_{2} \cdot \vec{n}\right)\left(\vec{S}_{2} \cdot \vec{v}_{2}\right)\left(\vec{v}_{2} \cdot \vec{n}\right) \\
& \left.+2 S_{1}^{2} S_{2}^{2}\left(1+\frac{5 v_{1}^{2}}{2}+\frac{5 v_{2}^{2}}{2}-5\left(\vec{v}_{1} \cdot \vec{n}\right)^{2}-5\left(\vec{v}_{2} \cdot \vec{n}\right)^{2}\right)\right]
\end{aligned}
$$




$$
\begin{aligned}
& +\frac{3 C_{1\left(\mathrm{ES}^{2}\right)} C_{2\left(\mathrm{ES}^{2}\right)} G}{2 m_{1} m_{2} r^{4}}\left[5\left(\vec{S}_{1} \cdot \vec{n}\right)^{2}\left(\vec{S}_{2} \cdot \vec{n}\right)\left(\dot{\vec{S}}_{2} \cdot \vec{v}_{2}\right)\right. \\
& -10\left(\vec{S}_{1} \cdot \vec{n}\right)^{2}\left(\dot{\vec{S}}_{2} \cdot \vec{S}_{2}\right)\left(\vec{v}_{2} \cdot \vec{n}\right)+5\left(\vec{S}_{1} \cdot \vec{n}\right)^{2}\left(\dot{\vec{S}}_{2} \cdot \vec{n}\right)\left(\vec{S}_{2} \cdot \vec{v}_{2}\right) \\
& +5\left(\vec{S}_{1} \cdot \vec{n}\right)^{2}\left(\vec{S}_{2} \cdot \vec{a}_{2}\right)\left(\vec{S}_{2} \cdot \vec{n}\right)+\left(\vec{S}_{1} \cdot \vec{n}\right)\left(\dot{\vec{S}}_{1} \cdot \vec{v}_{1}\right) S_{2}^{2}-5\left(\vec{S}_{1} \cdot \vec{n}\right)\left(\dot{\vec{S}}_{1} \cdot \vec{v}_{1}\right)\left(\vec{S}_{2} \cdot \vec{n}\right)^{2} \\
& -2\left(\vec{S}_{1} \cdot \vec{n}\right)\left(\vec{S}_{1} \cdot \dot{\vec{S}}_{2}\right)\left(\vec{S}_{2} \cdot \vec{v}_{2}\right)+4\left(\vec{S}_{1} \cdot \vec{n}\right)\left(\vec{S}_{1} \cdot \vec{v}_{2}\right)\left(\overrightarrow{\vec{S}}_{2} \cdot \vec{S}_{2}\right) \\
& -2\left(\vec{S}_{1} \cdot \vec{n}\right)\left(\vec{S}_{1} \cdot \vec{S}_{2}\right)\left(\dot{\vec{S}}_{2} \cdot \vec{v}_{2}\right)-5\left(\vec{S}_{1} \cdot \vec{n}\right)\left(\vec{S}_{1} \cdot \vec{a}_{1}\right)\left(\vec{S}_{2} \cdot \vec{n}\right)^{2}+\left(\vec{S}_{1} \cdot \vec{n}\right)\left(\vec{S}_{1} \cdot \vec{a}_{1}\right) S_{2}^{2} \\
& -2\left(\vec{S}_{1} \cdot \vec{n}\right)\left(\vec{S}_{1} \cdot \vec{S}_{2}\right)\left(\vec{S}_{2} \cdot \vec{a}_{2}\right)-S_{1}^{2}\left(\vec{S}_{2} \cdot \vec{a}_{2}\right)\left(\vec{S}_{2} \cdot \vec{n}\right)+2 S_{1}^{2}\left(\dot{\vec{S}}_{2} \cdot \vec{S}_{2}\right)\left(\vec{v}_{2} \cdot \vec{n}\right) \\
& +2\left(\vec{S}_{1} \cdot \vec{v}_{1}\right)\left(\dot{\vec{S}}_{1} \cdot \vec{S}_{2}\right)\left(\vec{S}_{2} \cdot \vec{n}\right)-5\left(\vec{S}_{1} \cdot \vec{v}_{1}\right)\left(\dot{\vec{S}}_{1} \cdot \vec{n}\right)\left(\vec{S}_{2} \cdot \vec{n}\right)^{2}+\left(\vec{S}_{1} \cdot \vec{v}_{1}\right)\left(\dot{\vec{S}}_{1} \cdot \vec{n}\right) S_{2}^{2} \\
& +2\left(\vec{S}_{1} \cdot \vec{a}_{1}\right)\left(\vec{S}_{1} \cdot \vec{S}_{2}\right)\left(\vec{S}_{2} \cdot \vec{n}\right)-S_{1}^{2}\left(\vec{S}_{2} \cdot \vec{n}\right)\left(\dot{\vec{S}}_{2} \cdot \vec{v}_{2}\right)-S_{1}^{2}\left(\dot{\vec{S}}_{2} \cdot \vec{n}\right)\left(\vec{S}_{2} \cdot \vec{v}_{2}\right) \\
& +2\left(\dot{\vec{S}}_{1} \cdot \vec{v}_{1}\right)\left(\vec{S}_{2} \cdot \vec{n}\right)\left(\vec{S}_{1} \cdot \vec{S}_{2}\right)+10\left(\overrightarrow{\vec{S}}_{1} \cdot \vec{S}_{1}\right)\left(\vec{S}_{2} \cdot \vec{n}\right)^{2}\left(\vec{v}_{1} \cdot \vec{n}\right) \\
& \left.-4\left(\dot{\vec{S}}_{1} \cdot \vec{S}_{1}\right)\left(\vec{S}_{2} \cdot \vec{n}\right)\left(\vec{S}_{2} \cdot \vec{v}_{1}\right)-2\left(\dot{\vec{S}}_{1} \cdot \vec{S}_{1}\right) S_{2}^{2}\left(\vec{v}_{1} \cdot \vec{n}\right)\right] \\
& -\frac{\left.C_{1\left(\mathrm{ES}^{2}\right)} C_{2(\mathrm{ES}}\right)}{m_{1} m_{2} r^{3}}\left[3\left(\vec{S}_{1} \cdot \vec{n}\right)^{2} \dot{S}_{2}^{2}+3\left(\vec{S}_{1} \cdot \vec{n}\right)^{2}\left(\ddot{\vec{S}}_{2} \cdot \vec{S}_{2}\right)-S_{1}^{2} \dot{S}_{2}^{2}-S_{1}^{2}\left(\ddot{\vec{S}}_{2} \cdot \vec{S}_{2}\right)\right. \\
& \left.+3 \dot{S}_{1}^{2}\left(\vec{S}_{2} \cdot \vec{n}\right)^{2}-\dot{S}_{1}^{2} S_{2}^{2}-\left(\ddot{\overrightarrow{S_{1}}} \cdot \vec{S}_{1}\right) S_{2}^{2}+3\left(\ddot{\vec{S}}_{1} \cdot \vec{S}_{1}\right)\left(\vec{S}_{2} \cdot \vec{n}\right)^{2}\right] \\
& 3 C_{1} C_{2}
\end{aligned}
$$

Fig. $2(\mathrm{a} 2)=\frac{3 C_{1\left(\mathrm{ES}^{2}\right)} C_{2\left(\mathrm{ES}^{2}\right)} G}{8 m_{1} m_{2} r^{5}}\left[5\left(\vec{S}_{1} \cdot \vec{n}\right)^{2} S_{2}^{2}\left(\vec{v}_{1} \cdot \vec{v}_{2}\right)-35\left(\vec{S}_{1} \cdot \vec{n}\right)^{2} S_{2}^{2}\left(\vec{v}_{1} \cdot \vec{n}\right)\left(\vec{v}_{2} \cdot \vec{n}\right)\right.$

$$
\begin{aligned}
& -315\left(\vec{S}_{1} \cdot \vec{n}\right)^{2}\left(\vec{S}_{2} \cdot \vec{n}\right)^{2}\left(\vec{v}_{1} \cdot \vec{n}\right)\left(\vec{v}_{2} \cdot \vec{n}\right)+70\left(\vec{S}_{1} \cdot \vec{n}\right)^{2}\left(\vec{S}_{2} \cdot \vec{n}\right)\left(\vec{S}_{2} \cdot \vec{v}_{1}\right)\left(\vec{v}_{2} \cdot \vec{n}\right) \\
& +70\left(\vec{S}_{1} \cdot \vec{n}\right)^{2}\left(\vec{S}_{2} \cdot \vec{n}\right)\left(\vec{S}_{2} \cdot \vec{v}_{2}\right)\left(\vec{v}_{1} \cdot \vec{n}\right)-10\left(\vec{S}_{1} \cdot \vec{n}\right)^{2}\left(\vec{S}_{2} \cdot \vec{v}_{1}\right)\left(\vec{S}_{2} \cdot \vec{v}_{2}\right) \\
& +35\left(\vec{S}_{1} \cdot \vec{n}\right)^{2}\left(\vec{S}_{2} \cdot \vec{n}\right)^{2}\left(\vec{v}_{1} \cdot \vec{v}_{2}\right)+10\left(\vec{S}_{1} \cdot \vec{n}\right)\left(\vec{S}_{1} \cdot \vec{v}_{1}\right) S_{2}^{2}\left(\vec{v}_{2} \cdot \vec{n}\right) \\
& +10\left(\vec{S}_{1} \cdot \vec{n}\right)\left(\vec{S}_{1} \cdot \vec{v}_{2}\right) S_{2}^{2}\left(\vec{v}_{1} \cdot \vec{n}\right)+140\left(\vec{S}_{1} \cdot \vec{n}\right)\left(\vec{S}_{1} \cdot \vec{S}_{2}\right)\left(\vec{S}_{2} \cdot \vec{n}\right)\left(\vec{v}_{1} \cdot \vec{n}\right)\left(\vec{v}_{2} \cdot \vec{n}\right) \\
& +70\left(\vec{S}_{1} \cdot \vec{n}\right)\left(\vec{S}_{1} \cdot \vec{v}_{1}\right)\left(\vec{S}_{2} \cdot \vec{n}\right)^{2}\left(\vec{v}_{2} \cdot \vec{n}\right)+70\left(\vec{S}_{1} \cdot \vec{n}\right)\left(\vec{S}_{1} \cdot \vec{v}_{2}\right)\left(\vec{S}_{2} \cdot \vec{n}\right)^{2}\left(\vec{v}_{1} \cdot \vec{n}\right) \\
& -20\left(\vec{S}_{1} \cdot \vec{n}\right)\left(\vec{S}_{1} \cdot \vec{S}_{2}\right)\left(\vec{S}_{2} \cdot \vec{v}_{1}\right)\left(\vec{v}_{2} \cdot \vec{n}\right)-20\left(\vec{S}_{1} \cdot \vec{n}\right)\left(\vec{S}_{1} \cdot \vec{v}_{2}\right)\left(\vec{S}_{2} \cdot \vec{n}\right)\left(\vec{S}_{2} \cdot \vec{v}_{1}\right) \\
& -20\left(\vec{S}_{1} \cdot \vec{n}\right)\left(\vec{S}_{1} \cdot \vec{S}_{2}\right)\left(\vec{S}_{2} \cdot \vec{v}_{2}\right)\left(\vec{v}_{1} \cdot \vec{n}\right)-20\left(\vec{S}_{1} \cdot \vec{n}\right)\left(\vec{S}_{1} \cdot \vec{v}_{1}\right)\left(\vec{S}_{2} \cdot \vec{n}\right)\left(\vec{S}_{2} \cdot \vec{v}_{2}\right) \\
& -20\left(\vec{S}_{1} \cdot \vec{n}\right)\left(\vec{S}_{1} \cdot \vec{S}_{2}\right)\left(\vec{S}_{2} \cdot \vec{n}\right)\left(\vec{v}_{1} \cdot \vec{v}_{2}\right)+4\left(\vec{S}_{1} \cdot \vec{v}_{1}\right)\left(\vec{S}_{1} \cdot \vec{S}_{2}\right)\left(\vec{S}_{2} \cdot \vec{v}_{2}\right) \\
& -20\left(\vec{S}_{1} \cdot \vec{v}_{1}\right)\left(\vec{S}_{1} \cdot \vec{S}_{2}\right)\left(\vec{S}_{2} \cdot \vec{n}\right)\left(\vec{v}_{2} \cdot \vec{n}\right)-10\left(\vec{S}_{1} \cdot \vec{v}_{1}\right)\left(\vec{S}_{1} \cdot \vec{v}_{2}\right)\left(\vec{S}_{2} \cdot \vec{n}\right)^{2} \\
& -2\left(\vec{S}_{1} \cdot \vec{v}_{1}\right)\left(\vec{S}_{1} \cdot \vec{v}_{2}\right) S_{2}^{2}-20\left(\vec{S}_{1} \cdot \vec{v}_{2}\right)\left(\vec{S}_{1} \cdot \vec{S}_{2}\right)\left(\vec{S}_{2} \cdot \vec{n}\right)\left(\vec{v}_{1} \cdot \vec{n}\right) \\
& +4\left(\vec{S}_{1} \cdot \vec{v}_{2}\right)\left(\vec{S}_{1} \cdot \vec{S}_{2}\right)\left(\vec{S}_{2} \cdot \vec{v}_{1}\right)-10\left(\vec{S}_{1} \cdot \vec{S}_{2}\right)^{2}\left(\vec{v}_{1} \cdot \vec{n}\right)\left(\vec{v}_{2} \cdot \vec{n}\right) \\
& +2\left(\vec{S}_{1} \cdot \vec{S}_{2}\right)^{2}\left(\vec{v}_{1} \cdot \vec{v}_{2}\right)-35 S_{1}^{2}\left(\vec{S}_{2} \cdot \vec{n}\right)^{2}\left(\vec{v}_{1} \cdot \vec{n}\right)\left(\vec{v}_{2} \cdot \vec{n}\right)+5 S_{1}^{2}\left(\vec{S}_{2} \cdot \vec{n}\right)^{2}\left(\vec{v}_{1} \cdot \vec{v}_{2}\right) \\
& +10 S_{1}^{2}\left(\vec{S}_{2} \cdot \vec{n}\right)\left(\vec{S}_{2} \cdot \vec{v}_{1}\right)\left(\vec{v}_{2} \cdot \vec{n}\right)+10 S_{1}^{2}\left(\vec{S}_{2} \cdot \vec{n}\right)\left(\vec{v}_{1} \cdot \vec{n}\right)\left(\vec{S}_{2} \cdot \vec{v}_{2}\right) \\
& \left.-2 S_{1}^{2}\left(\vec{S}_{2} \cdot \vec{v}_{1}\right)\left(\vec{S}_{2} \cdot \vec{v}_{2}\right)+15 S_{1}^{2} S_{2}^{2}\left(\vec{v}_{1} \cdot \vec{n}\right)\left(\vec{v}_{2} \cdot \vec{n}\right)-3 S_{1}^{2} S_{2}^{2}\left(\vec{v}_{1} \cdot \vec{v}_{2}\right)\right] \\
& +\frac{3 C_{1\left(\mathrm{ES}^{2}\right)} C_{2\left(\mathrm{ES}^{2}\right)} G}{4 m_{1} m_{2} r^{4}}\left[5\left(\vec{S}_{1} \cdot \vec{n}\right)^{2}\left(\vec{S}_{2} \cdot \vec{n}\right)\left(\dot{\vec{S}}_{2} \cdot \vec{v}_{1}\right)\right. \\
& -35\left(\vec{S}_{1} \cdot \vec{n}\right)^{2}\left(\vec{S}_{2} \cdot \vec{n}\right)\left(\dot{\vec{S}}_{2} \cdot \vec{n}\right)\left(\vec{v}_{1} \cdot \vec{n}\right)-5\left(\vec{S}_{1} \cdot \vec{n}\right)^{2}\left(\dot{\vec{S}}_{2} \cdot \vec{S}_{2}\right)\left(\vec{v}_{1} \cdot \vec{n}\right)
\end{aligned}
$$




$$
\begin{aligned}
& +5\left(\vec{S}_{1} \cdot \vec{n}\right)^{2}\left(\vec{S}_{2} \cdot \vec{v}_{1}\right)\left(\dot{\vec{S}}_{2} \cdot \vec{n}\right)+5\left(\vec{S}_{1} \cdot \vec{n}\right)\left(\dot{\vec{S}}_{1} \cdot \vec{n}\right) S_{2}^{2}\left(\vec{v}_{2} \cdot \vec{n}\right)-\left(\vec{S}_{1} \cdot \vec{n}\right)\left(\dot{\vec{S}}_{1} \cdot \vec{v}_{2}\right) S_{2}^{2} \\
& +35\left(\vec{S}_{1} \cdot \vec{n}\right)\left(\dot{\vec{S}}_{1} \cdot \vec{n}\right)\left(\vec{S}_{2} \cdot \vec{n}\right)^{2}\left(\vec{v}_{2} \cdot \vec{n}\right)-10\left(\vec{S}_{1} \cdot \vec{n}\right)\left(\dot{\vec{S}}_{1} \cdot \vec{S}_{2}\right)\left(\vec{S}_{2} \cdot \vec{n}\right)\left(\vec{v}_{2} \cdot \vec{n}\right) \\
& -5\left(\vec{S}_{1} \cdot \vec{n}\right)\left(\dot{\vec{S}}_{1} \cdot \vec{v}_{2}\right)\left(\vec{S}_{2} \cdot \vec{n}\right)^{2}+10\left(\vec{S}_{1} \cdot \vec{n}\right)\left(\vec{S}_{1} \cdot \dot{\vec{S}}_{2}\right)\left(\vec{S}_{2} \cdot \vec{n}\right)\left(\vec{v}_{1} \cdot \vec{n}\right) \\
& +10\left(\vec{S}_{1} \cdot \vec{n}\right)\left(\vec{S}_{1} \cdot \vec{S}_{2}\right)\left(\dot{\vec{S}}_{2} \cdot \vec{n}\right)\left(\vec{v}_{1} \cdot \vec{n}\right)-2\left(\vec{S}_{1} \cdot \vec{n}\right)\left(\vec{S}_{1} \cdot \vec{S}_{2}\right)\left(\dot{\vec{S}}_{2} \cdot \vec{v}_{1}\right) \\
& +10\left(\vec{S}_{1} \cdot \vec{n}\right)\left(\vec{S}_{1} \cdot \vec{v}_{1}\right)\left(\vec{S}_{2} \cdot \vec{n}\right)\left(\dot{\vec{S}}_{2} \cdot \vec{n}\right)+2\left(\vec{S}_{1} \cdot \vec{n}\right)\left(\vec{S}_{1} \cdot \vec{v}_{1}\right)\left(\dot{\vec{S}}_{2} \cdot \vec{S}_{2}\right) \\
& -2\left(\vec{S}_{1} \cdot \vec{n}\right)\left(\vec{S}_{1} \cdot \dot{\vec{S}}_{2}\right)\left(\vec{S}_{2} \cdot \vec{v}_{1}\right)-10\left(\vec{S}_{1} \cdot \vec{n}\right)\left(\dot{\vec{S}}_{1} \cdot \vec{n}\right)\left(\vec{S}_{2} \cdot \vec{n}\right)\left(\vec{S}_{2} \cdot \vec{v}_{2}\right) \\
& +2\left(\vec{S}_{1} \cdot \vec{n}\right)\left(\dot{\vec{S}}_{1} \cdot \vec{S}_{2}\right)\left(\vec{S}_{2} \cdot \vec{v}_{2}\right)-2\left(\vec{S}_{1} \cdot \vec{v}_{1}\right)\left(\vec{S}_{1} \cdot \dot{\vec{S}}_{2}\right)\left(\vec{S}_{2} \cdot \vec{n}\right) \\
& -2\left(\vec{S}_{1} \cdot \vec{v}_{1}\right)\left(\vec{S}_{1} \cdot \vec{S}_{2}\right)\left(\dot{\vec{S}}_{2} \cdot \vec{n}\right)-5\left(\vec{S}_{1} \cdot \vec{v}_{2}\right)\left(\dot{\vec{S}}_{1} \cdot \vec{n}\right)\left(\vec{S}_{2} \cdot \vec{n}\right)^{2} \\
& +2\left(\vec{S}_{1} \cdot \vec{v}_{2}\right)\left(\dot{\vec{S}}_{1} \cdot \vec{S}_{2}\right)\left(\vec{S}_{2} \cdot \vec{n}\right)-2\left(\vec{S}_{2} \cdot \vec{v}_{2}\right)\left(\dot{\vec{S}}_{1} \cdot \vec{S}_{1}\right)\left(\vec{S}_{2} \cdot \vec{n}\right) \\
& +2\left(\vec{S}_{2} \cdot \vec{v}_{2}\right)\left(\vec{S}_{1} \cdot \vec{S}_{2}\right)\left(\dot{\vec{S}}_{1} \cdot \vec{n}\right)-\left(\vec{S}_{1} \cdot \vec{v}_{2}\right)\left(\dot{\vec{S}}_{1} \cdot \vec{n}\right) S_{2}^{2} \\
& -10\left(\vec{S}_{1} \cdot \vec{S}_{2}\right)\left(\dot{\vec{S}}_{1} \cdot \vec{n}\right)\left(\vec{S}_{2} \cdot \vec{n}\right)\left(\vec{v}_{2} \cdot \vec{n}\right)+2\left(\vec{S}_{1} \cdot \vec{S}_{2}\right)\left(\dot{\vec{S}}_{1} \cdot \vec{S}_{2}\right)\left(\vec{v}_{2} \cdot \vec{n}\right) \\
& +2\left(\vec{S}_{1} \cdot \vec{S}_{2}\right)\left(\dot{\vec{S}}_{1} \cdot \vec{v}_{2}\right)\left(\vec{S}_{2} \cdot \vec{n}\right)-2\left(\vec{S}_{1} \cdot \vec{S}_{2}\right)\left(\vec{S}_{1} \cdot \dot{\vec{S}}_{2}\right)\left(\vec{v}_{1} \cdot \vec{n}\right) \\
& -5 S_{1}^{2}\left(\dot{\vec{S}}_{2} \cdot \vec{n}\right)\left(\vec{S}_{2} \cdot \vec{n}\right)\left(\vec{v}_{1} \cdot \vec{n}\right)+3 S_{1}^{2}\left(\dot{\vec{S}}_{2} \cdot \vec{S}_{2}\right)\left(\vec{v}_{1} \cdot \vec{n}\right)+S_{1}^{2}\left(\vec{S}_{2} \cdot \vec{n}\right)\left(\dot{\vec{S}}_{2} \cdot \vec{v}_{1}\right) \\
& \left.+S_{1}^{2}\left(\dot{\vec{S}}_{2} \cdot \vec{n}\right)\left(\vec{S}_{2} \cdot \vec{v}_{1}\right)-3\left(\dot{\vec{S}}_{1} \cdot \vec{S}_{1}\right) S_{2}^{2}\left(\vec{v}_{2} \cdot \vec{n}\right)+5\left(\dot{\vec{S}}_{1} \cdot \vec{S}_{1}\right)\left(\vec{S}_{2} \cdot \vec{n}\right)^{2}\left(\vec{v}_{2} \cdot \vec{n}\right)\right] \\
& +\frac{C_{1\left(\mathrm{ES}^{2}\right)} C_{2\left(\mathrm{ES}^{2}\right)} G}{2 m_{1} m_{2} r^{3}}\left[3\left(\vec{S}_{1} \cdot \vec{n}\right)\left(\dot{\vec{S}}_{1} \cdot \vec{n}\right)\left(\dot{\vec{S}}_{2} \cdot \vec{S}_{2}\right)-3\left(\vec{S}_{1} \cdot \vec{n}\right)\left(\dot{\vec{S}}_{1} \cdot \dot{\vec{S}}_{2}\right)\left(\vec{S}_{2} \cdot \vec{n}\right)\right. \\
& -3\left(\vec{S}_{1} \cdot \vec{n}\right)\left(\dot{\vec{S}}_{1} \cdot \vec{S}_{2}\right)\left(\dot{\vec{S}}_{2} \cdot \vec{n}\right)+15\left(\vec{S}_{1} \cdot \vec{n}\right)\left(\dot{\vec{S}}_{1} \cdot \vec{n}\right)\left(\vec{S}_{2} \cdot \vec{n}\right)\left(\dot{\vec{S}}_{2} \cdot \vec{n}\right) \\
& +\left(\vec{S}_{1} \cdot \vec{S}_{2}\right)\left(\dot{\vec{S}}_{1} \cdot \dot{\vec{S}}_{2}\right)-3\left(\vec{S}_{1} \cdot \vec{S}_{2}\right)\left(\dot{\vec{S}}_{1} \cdot \vec{n}\right)\left(\dot{\vec{S}}_{2} \cdot \vec{n}\right)+3\left(\dot{\vec{S}}_{1} \cdot \vec{S}_{1}\right)\left(\vec{S}_{2} \cdot \vec{n}\right)\left(\dot{\vec{S}}_{2} \cdot \vec{n}\right) \\
& \left.-3\left(\dot{\vec{S}}_{1} \cdot \vec{S}_{1}\right)\left(\dot{\vec{S}}_{2} \cdot \vec{S}_{2}\right)+\left(\vec{S}_{1} \cdot \dot{\vec{S}}_{2}\right)\left(\dot{\vec{S}}_{1} \cdot \vec{S}_{2}\right)-3\left(\vec{S}_{1} \cdot \dot{\vec{S}}_{2}\right)\left(\dot{\vec{S}}_{1} \cdot \vec{n}\right)\left(\vec{S}_{2} \cdot \vec{n}\right)\right] \text {, }
\end{aligned}
$$

Fig. $2(\mathrm{a} 3)=\frac{3 C_{1\left(\mathrm{ES}^{2}\right)} C_{2\left(\mathrm{ES}^{2}\right)} G}{m_{1} m_{2} r^{5}}\left[5\left(\vec{S}_{1} \cdot \vec{n}\right)^{2} S_{2}^{2}\left(\vec{v}_{1} \cdot \vec{v}_{2}\right)-35\left(\vec{S}_{1} \cdot \vec{n}\right)^{2}\left(\vec{S}_{2} \cdot \vec{n}\right)^{2}\right.$

$$
\begin{aligned}
& \left.+20\left(\vec{S}_{1} \cdot \vec{n}\right)\left(\vec{S}_{1} \cdot \vec{S}_{2}\right)\left(\vec{S}_{2} \cdot \vec{n}\right)-S_{1}^{2} S_{2}^{2}+5 S_{1}^{2}\left(\vec{S}_{2} \cdot \vec{n}\right)^{2}-2\left(\vec{S}_{1} \cdot \vec{S}_{2}\right)^{2}\right]\left(\vec{v}_{1} \cdot \vec{v}_{2}\right) \\
& +\frac{3 C_{1\left(\mathrm{ES}^{2}\right)} C_{2\left(\mathrm{ES}^{2}\right)} G}{m_{1} m_{2} r^{4}}\left[10\left(\vec{S}_{1} \cdot \vec{n}\right)^{2}\left(\dot{\vec{S}}_{2} \cdot \vec{S}_{2}\right)\left(\vec{v}_{1} \cdot \vec{n}\right)-5\left(\vec{S}_{1} \cdot \vec{n}\right)^{2}\left(\vec{S}_{2} \cdot \vec{v}_{1}\right)\left(\dot{\vec{S}}_{2} \cdot \vec{n}\right)\right. \\
& -5\left(\vec{S}_{1} \cdot \vec{n}\right)^{2}\left(\vec{S}_{2} \cdot \vec{n}\right)\left(\dot{\vec{S}}_{2} \cdot \vec{v}_{1}\right)-\left(\vec{S}_{1} \cdot \vec{n}\right)\left(\dot{\vec{S}}_{1} \cdot \vec{v}_{2}\right) S_{2}^{2} \\
& -4\left(\vec{S}_{1} \cdot \vec{n}\right)\left(\vec{S}_{1} \cdot \vec{v}_{1}\right)\left(\dot{\vec{S}}_{2} \cdot \vec{S}_{2}\right)+5\left(\vec{S}_{1} \cdot \vec{n}\right)\left(\dot{\vec{S}}_{1} \cdot \vec{v}_{2}\right)\left(\vec{S}_{2} \cdot \vec{n}\right)^{2} \\
& +2\left(\vec{S}_{1} \cdot \vec{n}\right)\left(\vec{S}_{1} \cdot \vec{S}_{2}\right)\left(\overrightarrow{\vec{S}}_{2} \cdot \vec{v}_{1}\right)+2\left(\vec{S}_{1} \cdot \vec{n}\right)\left(\vec{S}_{1} \cdot \dot{\vec{S}}_{2}\right)\left(\vec{S}_{2} \cdot \vec{v}_{1}\right) \\
& -2\left(\vec{S}_{1} \cdot \vec{v}_{2}\right)\left(\dot{\vec{S}}_{1} \cdot \vec{S}_{2}\right)\left(\vec{S}_{2} \cdot \vec{n}\right)-\left(\vec{S}_{1} \cdot \vec{v}_{2}\right)\left(\dot{\vec{S}}_{1} \cdot \vec{n}\right) S_{2}^{2} \\
& +5\left(\vec{S}_{1} \cdot \vec{v}_{2}\right)\left(\dot{\vec{S}}_{1} \cdot \vec{n}\right)\left(\vec{S}_{2} \cdot \vec{n}\right)^{2}-2\left(\vec{S}_{1} \cdot \vec{S}_{2}\right)\left(\dot{\vec{S}}_{1} \cdot \vec{v}_{2}\right)\left(\vec{S}_{2} \cdot \vec{n}\right)+S_{1}^{2}\left(\vec{S}_{2} \cdot \vec{v}_{1}\right)\left(\dot{\vec{S}}_{2} \cdot \vec{n}\right) \\
& -2 S_{1}^{2}\left(\dot{\vec{S}}_{2} \cdot \vec{S}_{2}\right)\left(\vec{v}_{1} \cdot \vec{n}\right)+S_{1}^{2}\left(\vec{S}_{2} \cdot \vec{n}\right)\left(\dot{\vec{S}}_{2} \cdot \vec{v}_{1}\right)+4\left(\dot{\vec{S}}_{1} \cdot \vec{S}_{1}\right)\left(\vec{S}_{2} \cdot \vec{n}\right)\left(\vec{S}_{2} \cdot \vec{v}_{2}\right) \\
& \left.-10\left(\dot{\vec{S}}_{1} \cdot \vec{S}_{1}\right)\left(\vec{S}_{2} \cdot \vec{n}\right)^{2}\left(\vec{v}_{2} \cdot \vec{n}\right)+2\left(\dot{\vec{S}}_{1} \cdot \vec{S}_{1}\right) S_{2}^{2}\left(\vec{v}_{2} \cdot \vec{n}\right)\right]
\end{aligned}
$$




$$
\begin{aligned}
& +\frac{C_{1\left(\mathrm{ES}^{2}\right)} C_{2\left(\mathrm{ES}^{2}\right)} G}{m_{1} m_{2} r^{3}}\left[3\left(\vec{S}_{1} \cdot \vec{n}\right)\left(\dot{\vec{S}}_{1} \cdot \dot{\vec{S}}_{2}\right)\left(\vec{S}_{2} \cdot \vec{n}\right)\right. \\
& -12\left(\vec{S}_{1} \cdot \vec{n}\right)\left(\dot{\vec{S}}_{1} \cdot \vec{n}\right)\left(\dot{\vec{S}}_{2} \cdot \vec{S}_{2}\right)+3\left(\vec{S}_{1} \cdot \vec{n}\right)\left(\dot{\vec{S}}_{1} \cdot \vec{S}_{2}\right)\left(\dot{\vec{S}}_{2} \cdot \vec{n}\right) \\
& +3\left(\vec{S}_{1} \cdot \vec{S}_{2}\right)\left(\dot{\vec{S}}_{1} \cdot \vec{n}\right)\left(\dot{\vec{S}}_{2} \cdot \vec{n}\right)-2\left(\vec{S}_{1} \cdot \vec{S}_{2}\right)\left(\dot{\vec{S}}_{1} \cdot \dot{\vec{S}}_{2}\right)+8\left(\dot{\vec{S}}_{1} \cdot \vec{S}_{1}\right)\left(\dot{\vec{S}}_{2} \cdot \vec{S}_{2}\right) \\
& -12\left(\dot{\vec{S}}_{1} \cdot \vec{S}_{1}\right)\left(\dot{\vec{S}}_{2} \cdot \vec{n}\right)\left(\vec{S}_{2} \cdot \vec{n}\right)-2\left(\dot{\vec{S}}_{1} \cdot \vec{S}_{2}\right)\left(\dot{\vec{S}}_{2} \cdot \vec{S}_{1}\right) \\
& \left.+3\left(\vec{S}_{1} \cdot \dot{\vec{S}}_{2}\right)\left(\dot{\vec{S}}_{1} \cdot \vec{n}\right)\left(\vec{S}_{2} \cdot \vec{n}\right)\right] \text {, } \\
& \text { Fig. } 2 \text { (b1) }=\frac{C_{1\left(\mathrm{BS}^{3}\right)}}{4} \frac{G}{m_{1}^{2} r^{5}}\left[70\left(\vec{S}_{1} \cdot \vec{n}\right)^{3}\left(\vec{S}_{2} \cdot \vec{n}\right)\left(1+\frac{3 v_{1}^{2}}{2}+\frac{3 v_{2}^{2}}{2}\right)\right. \\
& -30\left(\vec{S}_{1} \cdot \vec{n}\right)^{2}\left(\vec{S}_{1} \cdot \vec{S}_{2}\right)\left(1+\frac{13 v_{1}^{2}}{6}+\frac{13 v_{2}^{2}}{6}-\frac{14\left(\vec{v}_{1} \cdot \vec{n}\right)^{2}}{3}-\frac{14\left(\vec{v}_{2} \cdot \vec{n}\right)^{2}}{3}\right) \\
& +6 S_{1}^{2}\left(\vec{S}_{1} \cdot \vec{S}_{2}\right)\left(1+\frac{13 v_{1}^{2}}{6}+\frac{13 v_{2}^{2}}{6}-\frac{10\left(\vec{v}_{1} \cdot \vec{n}\right)^{2}}{3}-\frac{10\left(\vec{v}_{2} \cdot \vec{n}\right)^{2}}{3}\right) \\
& -30 S_{1}^{2}\left(\vec{S}_{1} \cdot \vec{n}\right)\left(\vec{S}_{2} \cdot \vec{n}\right)\left(1+\frac{3 v_{1}^{2}}{2}+\frac{3 v_{2}^{2}}{2}\right)-2\left(\vec{S}_{1} \cdot \vec{v}_{1}\right)^{2}\left(\vec{S}_{1} \cdot \vec{S}_{2}\right) \\
& -140\left(\vec{S}_{1} \cdot \vec{n}\right)^{3}\left(\vec{S}_{2} \cdot \vec{v}_{1}\right)\left(\vec{v}_{1} \cdot \vec{n}\right)-105\left(\vec{S}_{1} \cdot \vec{n}\right)^{3}\left(\vec{S}_{2} \cdot \vec{v}_{2}\right)\left(\vec{v}_{2} \cdot \vec{n}\right) \\
& -140\left(\vec{S}_{1} \cdot \vec{n}\right)^{2}\left(\vec{S}_{1} \cdot \vec{v}_{2}\right)\left(\vec{S}_{2} \cdot \vec{n}\right)\left(\vec{v}_{2} \cdot \vec{n}\right)+85\left(\vec{S}_{1} \cdot \vec{n}\right)^{2}\left(\vec{S}_{1} \cdot \vec{v}_{1}\right)\left(\vec{S}_{2} \cdot \vec{v}_{1}\right) \\
& -175\left(\vec{S}_{1} \cdot \vec{n}\right)^{2}\left(\vec{S}_{1} \cdot \vec{v}_{1}\right)\left(\vec{S}_{2} \cdot \vec{n}\right)\left(\vec{v}_{1} \cdot \vec{n}\right)+65\left(\vec{S}_{1} \cdot \vec{n}\right)^{2}\left(\vec{S}_{1} \cdot \vec{v}_{2}\right)\left(\vec{S}_{2} \cdot \vec{v}_{2}\right) \\
& +50\left(\vec{S}_{1} \cdot \vec{n}\right)\left(\vec{S}_{1} \cdot \vec{v}_{1}\right)^{2}\left(\vec{S}_{2} \cdot \vec{n}\right)+40\left(\vec{S}_{1} \cdot \vec{n}\right)\left(\vec{S}_{1} \cdot \vec{v}_{2}\right)^{2}\left(\vec{S}_{2} \cdot \vec{n}\right) \\
& -30\left(\vec{S}_{1} \cdot \vec{n}\right)\left(\vec{S}_{1} \cdot \vec{v}_{1}\right)\left(\vec{S}_{1} \cdot \vec{S}_{2}\right)\left(\vec{v}_{1} \cdot \vec{n}\right)-40\left(\vec{S}_{1} \cdot \vec{n}\right)\left(\vec{S}_{1} \cdot \vec{v}_{2}\right)\left(\vec{S}_{1} \cdot \vec{S}_{2}\right)\left(\vec{v}_{2} \cdot \vec{n}\right) \\
& +60 S_{1}^{2}\left(\vec{S}_{1} \cdot \vec{n}\right)\left(\vec{S}_{2} \cdot \vec{v}_{1}\right)\left(\vec{v}_{1} \cdot \vec{n}\right)+45 S_{1}^{2}\left(\vec{S}_{1} \cdot \vec{n}\right)\left(\vec{S}_{2} \cdot \vec{v}_{2}\right)\left(\vec{v}_{2} \cdot \vec{n}\right) \\
& +25 S_{1}^{2}\left(\vec{S}_{2} \cdot \vec{n}\right)\left(\vec{S}_{1} \cdot \vec{v}_{1}\right)\left(\vec{v}_{1} \cdot \vec{n}\right)-13 S_{1}^{2}\left(\vec{S}_{1} \cdot \vec{v}_{2}\right)\left(\vec{S}_{2} \cdot \vec{v}_{2}\right) \\
& \left.+20 S_{1}^{2}\left(\vec{S}_{2} \cdot \vec{n}\right)\left(\vec{S}_{1} \cdot \vec{v}_{2}\right)\left(\vec{v}_{2} \cdot \vec{n}\right)-17 S_{1}^{2}\left(\vec{S}_{1} \cdot \vec{v}_{1}\right)\left(\vec{S}_{2} \cdot \vec{v}_{1}\right)\right] \\
& +\frac{C_{1\left(\mathrm{BS}^{3}\right)}}{2} \frac{G}{m_{1}^{2} r^{4}}\left[10\left(\vec{S}_{1} \cdot \vec{n}\right)^{3}\left(\vec{S}_{2} \cdot \vec{a}_{1}\right)\right. \\
& -10\left(\vec{S}_{1} \cdot \vec{n}\right)^{2}\left(\vec{S}_{1} \cdot \vec{a}_{2}\right)\left(\vec{S}_{2} \cdot \vec{n}\right)-10\left(\vec{S}_{1} \cdot \vec{n}\right)^{2}\left(\dot{\vec{S}}_{1} \cdot \vec{S}_{2}\right)\left(\vec{v}_{1} \cdot \vec{n}\right) \\
& +10\left(\vec{S}_{1} \cdot \vec{n}\right)^{2}\left(\vec{S}_{1} \cdot \dot{\vec{S}}_{2}\right)\left(\vec{v}_{2} \cdot \vec{n}\right)-10\left(\vec{S}_{1} \cdot \vec{n}\right)^{2}\left(\vec{S}_{1} \cdot \vec{S}_{2}\right)\left(\vec{a}_{1} \cdot \vec{n}\right) \\
& +10\left(\vec{S}_{1} \cdot \vec{n}\right)^{2}\left(\vec{S}_{1} \cdot \vec{S}_{2}\right)\left(\vec{a}_{2} \cdot \vec{n}\right)-10\left(\vec{S}_{1} \cdot \vec{n}\right)^{2}\left(\vec{S}_{1} \cdot \vec{v}_{2}\right)\left(\dot{\vec{S}}_{2} \cdot \vec{n}\right) \\
& +30\left(\vec{S}_{1} \cdot \vec{n}\right)^{2}\left(\dot{\vec{S}}_{1} \cdot \vec{n}\right)\left(\vec{S}_{2} \cdot \vec{v}_{1}\right)+10\left(\vec{S}_{1} \cdot \vec{n}\right)\left(\dot{\vec{S}}_{1} \cdot \vec{S}_{1}\right)\left(\vec{S}_{2} \cdot \vec{n}\right)\left(\vec{v}_{1} \cdot \vec{n}\right) \\
& +5 S_{1}^{2}\left(\vec{S}_{1} \cdot \vec{n}\right)\left(\vec{S}_{2} \cdot \vec{n}\right)\left(\vec{a}_{1} \cdot \vec{n}\right)-7 S_{1}^{2}\left(\vec{S}_{1} \cdot \vec{n}\right)\left(\vec{S}_{2} \cdot \vec{a}_{1}\right) \\
& -20\left(\vec{S}_{1} \cdot \vec{n}\right)\left(\dot{\vec{S}}_{1} \cdot \vec{n}\right)\left(\vec{S}_{1} \cdot \vec{S}_{2}\right)\left(\vec{v}_{1} \cdot \vec{n}\right)+4\left(\vec{S}_{1} \cdot \vec{n}\right)\left(\vec{S}_{1} \cdot \vec{a}_{1}\right)\left(\vec{S}_{1} \cdot \vec{S}_{2}\right) \\
& +4\left(\vec{S}_{1} \cdot \vec{n}\right)\left(\dot{\vec{S}}_{1} \cdot \vec{v}_{1}\right)\left(\vec{S}_{1} \cdot \vec{S}_{2}\right)+4\left(\vec{S}_{1} \cdot \vec{n}\right)\left(\vec{S}_{1} \cdot \vec{v}_{1}\right)\left(\dot{\vec{S}}_{1} \cdot \vec{S}_{2}\right) \\
& -14\left(\vec{S}_{1} \cdot \vec{n}\right)\left(\dot{\vec{S}}_{1} \cdot \vec{S}_{1}\right)\left(\vec{S}_{2} \cdot \vec{v}_{1}\right)+5 S_{1}^{2}\left(\dot{\vec{S}}_{1} \cdot \vec{n}\right)\left(\vec{S}_{2} \cdot \vec{n}\right)\left(\vec{v}_{1} \cdot \vec{n}\right) \\
& +2 S_{1}^{2}\left(\vec{S}_{1} \cdot \vec{a}_{2}\right)\left(\vec{S}_{2} \cdot \vec{n}\right)-S_{1}^{2}\left(\vec{S}_{1} \cdot \vec{a}_{1}\right)\left(\vec{S}_{2} \cdot \vec{n}\right)+4\left(\dot{\vec{S}}_{1} \cdot \vec{n}\right)\left(\vec{S}_{1} \cdot \vec{v}_{1}\right)\left(\vec{S}_{1} \cdot \vec{S}_{2}\right) \\
& -2 S_{1}^{2}\left(\vec{S}_{1} \cdot \dot{\vec{S}}_{2}\right)\left(\vec{v}_{2} \cdot \vec{n}\right)+2\left(\vec{S}_{1} \cdot \dot{\vec{S}}_{1}\right)\left(\vec{S}_{1} \cdot \vec{S}_{2}\right)\left(\vec{v}_{1} \cdot \vec{n}\right)+S_{1}^{2}\left(\vec{S}_{1} \cdot \vec{S}_{2}\right)\left(\vec{a}_{1} \cdot \vec{n}\right)
\end{aligned}
$$




$$
\begin{aligned}
& -2 S_{1}^{2}\left(\vec{S}_{1} \cdot \vec{S}_{2}\right)\left(\vec{a}_{2} \cdot \vec{n}\right)-2\left(\vec{S}_{1} \cdot \dot{\vec{S}}_{1}\right)\left(\vec{S}_{1} \cdot \vec{v}_{1}\right)\left(\vec{S}_{2} \cdot \vec{n}\right)-S_{1}^{2}\left(\dot{\vec{S}}_{1} \cdot \vec{v}_{1}\right)\left(\vec{S}_{2} \cdot \vec{n}\right) \\
& \left.+S_{1}^{2}\left(\overrightarrow{\vec{S}}_{1} \cdot \vec{S}_{2}\right)\left(\vec{v}_{1} \cdot \vec{n}\right)+2 S_{1}^{2}\left(\vec{S}_{1} \cdot \vec{v}_{2}\right)\left(\dot{\vec{S}}_{2} \cdot \vec{n}\right)-7 S_{1}^{2}\left(\dot{\vec{S}}_{1} \cdot \vec{n}\right)\left(\vec{S}_{2} \cdot \vec{v}_{1}\right)\right],
\end{aligned}
$$

Fig. 2(b2) $=\frac{C_{1\left(\mathrm{BS}^{3}\right)}}{4} \frac{G}{m_{1}^{2} r^{5}}\left[35\left(\vec{S}_{1} \cdot \vec{n}\right)^{3}\left(\vec{S}_{2} \cdot \vec{v}_{1}\right)\left(\vec{v}_{2} \cdot \vec{n}\right)+35\left(\vec{S}_{1} \cdot \vec{n}\right)^{3}\left(\vec{S}_{2} \cdot \vec{v}_{2}\right)\left(\vec{v}_{1} \cdot \vec{n}\right)\right.$

$+35\left(\vec{S}_{1} \cdot \vec{n}\right)^{3}\left(\vec{S}_{2} \cdot \vec{n}\right)\left(\vec{v}_{1} \cdot \vec{v}_{2}\right)-315\left(\vec{S}_{1} \cdot \vec{n}\right)^{3}\left(\vec{S}_{2} \cdot \vec{n}\right)\left(\vec{v}_{1} \cdot \vec{n}\right)\left(\vec{v}_{2} \cdot \vec{n}\right)$

$+35\left(\vec{S}_{1} \cdot \vec{n}\right)^{2}\left(\vec{S}_{1} \cdot \vec{S}_{2}\right)\left(\vec{v}_{1} \cdot \vec{n}\right)\left(\vec{v}_{2} \cdot \vec{n}\right)+105\left(\vec{S}_{1} \cdot \vec{n}\right)^{2}\left(\vec{S}_{2} \cdot \vec{n}\right)\left(\vec{v}_{2} \cdot \vec{n}\right)\left(\vec{S}_{1} \cdot \vec{v}_{1}\right)$

$+105\left(\vec{S}_{1} \cdot \vec{n}\right)^{2}\left(\vec{S}_{1} \cdot \vec{v}_{2}\right)\left(\vec{S}_{2} \cdot \vec{n}\right)\left(\vec{v}_{1} \cdot \vec{n}\right)-15\left(\vec{S}_{1} \cdot \vec{n}\right)^{2}\left(\vec{S}_{1} \cdot \vec{v}_{2}\right)\left(\vec{S}_{2} \cdot \vec{v}_{1}\right)$

$-15\left(\vec{S}_{1} \cdot \vec{n}\right)^{2}\left(\vec{S}_{1} \cdot \vec{v}_{1}\right)\left(\vec{S}_{2} \cdot \vec{v}_{2}\right)-5\left(\vec{S}_{1} \cdot \vec{n}\right)^{2}\left(\vec{S}_{1} \cdot \vec{S}_{2}\right)\left(\vec{v}_{1} \cdot \vec{v}_{2}\right)$

$-10\left(\vec{S}_{1} \cdot \vec{n}\right)\left(\vec{S}_{1} \cdot \vec{v}_{1}\right)\left(\vec{S}_{1} \cdot \vec{S}_{2}\right)\left(\vec{v}_{2} \cdot \vec{n}\right)-30\left(\vec{S}_{1} \cdot \vec{n}\right)\left(\vec{S}_{1} \cdot \vec{v}_{1}\right)\left(\vec{S}_{1} \cdot \vec{v}_{2}\right)\left(\vec{S}_{2} \cdot \vec{n}\right)$

$-10\left(\vec{S}_{1} \cdot \vec{n}\right)\left(\vec{S}_{1} \cdot \vec{v}_{2}\right)\left(\vec{S}_{1} \cdot \vec{S}_{2}\right)\left(\vec{v}_{1} \cdot \vec{n}\right)+2\left(\vec{S}_{1} \cdot \vec{v}_{1}\right)\left(\vec{S}_{1} \cdot \vec{v}_{2}\right)\left(\vec{S}_{1} \cdot \vec{S}_{2}\right)$

$-15 S_{1}^{2}\left(\vec{S}_{1} \cdot \vec{n}\right)\left(\vec{S}_{2} \cdot \vec{v}_{1}\right)\left(\vec{v}_{2} \cdot \vec{n}\right)-15 S_{1}^{2}\left(\vec{S}_{1} \cdot \vec{n}\right)\left(\vec{S}_{2} \cdot \vec{v}_{2}\right)\left(\vec{v}_{1} \cdot \vec{n}\right)$

$-15 S_{1}^{2}\left(\vec{S}_{1} \cdot \vec{n}\right)\left(\vec{S}_{2} \cdot \vec{n}\right)\left(\vec{v}_{1} \cdot \vec{v}_{2}\right)+105 S_{1}^{2}\left(\vec{S}_{1} \cdot \vec{n}\right)\left(\vec{S}_{2} \cdot \vec{n}\right)\left(\vec{v}_{1} \cdot \vec{n}\right)\left(\vec{v}_{2} \cdot \vec{n}\right)$

$-15 S_{1}^{2}\left(\vec{S}_{1} \cdot \vec{v}_{1}\right)\left(\vec{S}_{2} \cdot \vec{n}\right)\left(\vec{v}_{2} \cdot \vec{n}\right)-15 S_{1}^{2}\left(\vec{S}_{1} \cdot \vec{v}_{2}\right)\left(\vec{S}_{2} \cdot \vec{n}\right)\left(\vec{v}_{1} \cdot \vec{n}\right)$

$+3 S_{1}^{2}\left(\vec{S}_{1} \cdot \vec{v}_{2}\right)\left(\vec{S}_{2} \cdot \vec{v}_{1}\right)-5 S_{1}^{2}\left(\vec{S}_{1} \cdot \vec{S}_{2}\right)\left(\vec{v}_{1} \cdot \vec{n}\right)\left(\vec{v}_{2} \cdot \vec{n}\right)$

$\left.+3 S_{1}^{2}\left(\vec{S}_{1} \cdot \vec{v}_{1}\right)\left(\vec{S}_{2} \cdot \vec{v}_{2}\right)+S_{1}^{2}\left(\vec{S}_{1} \cdot \vec{S}_{2}\right)\left(\vec{v}_{1} \cdot \vec{v}_{2}\right)\right]$

$+\frac{C_{1\left(\mathrm{BS}^{3}\right)}}{4} \frac{G}{m_{1}^{2} r^{4}}\left[5\left(\vec{S}_{1} \cdot \vec{n}\right)^{3}\left(\dot{\vec{S}}_{2} \cdot \vec{v}_{1}\right)-35\left(\vec{S}_{1} \cdot \vec{n}\right)^{3}\left(\dot{\vec{S}}_{2} \cdot \vec{n}\right)\left(\vec{v}_{1} \cdot \vec{n}\right)\right.$

$+105\left(\vec{S}_{1} \cdot \vec{n}\right)^{2}\left(\dot{\vec{S}}_{1} \cdot \vec{n}\right)\left(\vec{S}_{2} \cdot \vec{n}\right)\left(\vec{v}_{2} \cdot \vec{n}\right)-5\left(\vec{S}_{1} \cdot \vec{n}\right)^{2}\left(\dot{\vec{S}}_{1} \cdot \vec{S}_{2}\right)\left(\vec{v}_{2} \cdot \vec{n}\right)$

$-15\left(\vec{S}_{1} \cdot \vec{n}\right)^{2}\left(\dot{\vec{S}}_{1} \cdot \vec{v}_{2}\right)\left(\vec{S}_{2} \cdot \vec{n}\right)+5\left(\vec{S}_{1} \cdot \vec{n}\right)^{2}\left(\vec{S}_{1} \cdot \dot{\vec{S}}_{2}\right)\left(\vec{v}_{1} \cdot \vec{n}\right)$

$+15\left(\vec{S}_{1} \cdot \vec{n}\right)^{2}\left(\vec{S}_{1} \cdot \vec{v}_{1}\right)\left(\dot{\vec{S}}_{2} \cdot \vec{n}\right)-15\left(\vec{S}_{1} \cdot \vec{n}\right)^{2}\left(\dot{\vec{S}}_{1} \cdot \vec{n}\right)\left(\vec{S}_{2} \cdot \vec{v}_{2}\right)$

$-30\left(\vec{S}_{1} \cdot \vec{n}\right)\left(\dot{\vec{S}}_{1} \cdot \vec{S}_{1}\right)\left(\vec{S}_{2} \cdot \vec{n}\right)\left(\vec{v}_{2} \cdot \vec{n}\right)-10\left(\vec{S}_{1} \cdot \vec{n}\right)\left(\dot{\vec{S}}_{1} \cdot \vec{n}\right)\left(\vec{S}_{1} \cdot \vec{S}_{2}\right)\left(\vec{v}_{2} \cdot \vec{n}\right)$

$+2\left(\vec{S}_{1} \cdot \vec{n}\right)\left(\dot{\vec{S}}_{1} \cdot \vec{v}_{2}\right)\left(\vec{S}_{1} \cdot \vec{S}_{2}\right)-2\left(\vec{S}_{1} \cdot \vec{n}\right)\left(\vec{S}_{1} \cdot \vec{v}_{1}\right)\left(\vec{S}_{1} \cdot \dot{\vec{S}}_{2}\right)$

$-30\left(\vec{S}_{1} \cdot \vec{n}\right)\left(\vec{S}_{1} \cdot \vec{v}_{2}\right)\left(\dot{\vec{S}}_{1} \cdot \vec{n}\right)\left(\vec{S}_{2} \cdot \vec{n}\right)+2\left(\vec{S}_{1} \cdot \vec{n}\right)\left(\vec{S}_{1} \cdot \vec{v}_{2}\right)\left(\dot{\vec{S}}_{1} \cdot \vec{S}_{2}\right)$

$+6\left(\vec{S}_{1} \cdot \vec{n}\right)\left(\dot{\vec{S}}_{1} \cdot \vec{S}_{1}\right)\left(\vec{S}_{2} \cdot \vec{v}_{2}\right)-15 S_{1}^{2}\left(\dot{\vec{S}}_{1} \cdot \vec{n}\right)\left(\vec{S}_{2} \cdot \vec{n}\right)\left(\vec{v}_{2} \cdot \vec{n}\right)$

$-3 S_{1}^{2}\left(\vec{S}_{1} \cdot \vec{n}\right)\left(\dot{\vec{S}}_{2} \cdot \vec{v}_{1}\right)+15 S_{1}^{2}\left(\vec{S}_{1} \cdot \vec{n}\right)\left(\dot{\vec{S}}_{2} \cdot \vec{n}\right)\left(\vec{v}_{1} \cdot \vec{n}\right)$

$+S_{1}^{2}\left(\dot{\vec{S}}_{1} \cdot \vec{S}_{2}\right)\left(\vec{v}_{2} \cdot \vec{n}\right)+3 S_{1}^{2}\left(\dot{\vec{S}}_{1} \cdot \vec{v}_{2}\right)\left(\vec{S}_{2} \cdot \vec{n}\right)-S_{1}^{2}\left(\vec{S}_{1} \cdot \dot{\vec{S}}_{2}\right)\left(\vec{v}_{1} \cdot \vec{n}\right)$

$-3 S_{1}^{2}\left(\vec{S}_{1} \cdot \vec{v}_{1}\right)\left(\dot{\vec{S}}_{2} \cdot \vec{n}\right)+3 S_{1}^{2}\left(\dot{\vec{S}}_{1} \cdot \vec{n}\right)\left(\vec{S}_{2} \cdot \vec{v}_{2}\right)+2\left(\dot{\vec{S}}_{1} \cdot \vec{S}_{1}\right)\left(\vec{S}_{1} \cdot \vec{S}_{2}\right)\left(\vec{v}_{2} \cdot \vec{n}\right)$

$\left.+6\left(\vec{S}_{1} \cdot \vec{v}_{2}\right)\left(\dot{\vec{S}}_{1} \cdot \vec{S}_{1}\right)\left(\vec{S}_{2} \cdot \vec{n}\right)+2\left(\vec{S}_{1} \cdot \vec{v}_{2}\right)\left(\dot{\vec{S}}_{1} \cdot \vec{n}\right)\left(\vec{S}_{1} \cdot \vec{S}_{2}\right)\right]$

$+\frac{C_{1\left(\mathrm{BS}^{3}\right)}}{12} \frac{G}{m_{1}^{2} r^{3}}\left[45\left(\vec{S}_{1} \cdot \vec{n}\right)^{2}\left(\dot{\vec{S}}_{1} \cdot \vec{n}\right)\left(\dot{\vec{S}}_{2} \cdot \vec{n}\right)-3\left(\vec{S}_{1} \cdot \vec{n}\right)^{2}\left(\dot{\vec{S}}_{1} \cdot \dot{\vec{S}}_{2}\right)\right.$

$-18\left(\vec{S}_{1} \cdot \vec{n}\right)\left(\dot{\vec{S}}_{1} \cdot \vec{S}_{1}\right)\left(\dot{\vec{S}}_{2} \cdot \vec{n}\right)-6\left(\vec{S}_{1} \cdot \vec{n}\right)\left(\dot{\vec{S}}_{1} \cdot \vec{n}\right)\left(\vec{S}_{1} \cdot \dot{\vec{S}}_{2}\right)$

$\left.+2\left(\dot{\vec{S}}_{1} \cdot \vec{S}_{1}\right)\left(\vec{S}_{1} \cdot \dot{\vec{S}}_{2}\right)-9 S_{1}^{2}\left(\dot{\vec{S}}_{1} \cdot \vec{n}\right)\left(\dot{\vec{S}}_{2} \cdot \vec{n}\right)+S_{1}^{2}\left(\dot{\vec{S}}_{1} \cdot \dot{\vec{S}}_{2}\right)\right]$, 
Fig. $2(\mathrm{~b} 3)=2 C_{1\left(\mathrm{BS}^{3}\right)} \frac{G}{m_{1}^{2} r^{5}}\left\{35\left(\vec{S}_{1} \cdot \vec{n}\right)^{3}\left(\vec{S}_{2} \cdot \vec{v}_{1}\right)\left(\vec{v}_{2} \cdot \vec{n}\right)-35\left(\vec{S}_{1} \cdot \vec{n}\right)^{3}\left(\vec{S}_{2} \cdot \vec{n}\right)\left(\vec{v}_{1} \cdot \vec{v}_{2}\right)\right.$

$$
\begin{aligned}
& -35\left(\vec{S}_{1} \cdot \vec{S}_{2}\right)\left(\vec{S}_{1} \cdot \vec{n}\right)^{2}\left(\vec{v}_{1} \cdot \vec{n}\right)\left(\vec{v}_{2} \cdot \vec{n}\right)+35\left(\vec{S}_{1} \cdot \vec{n}\right)^{2}\left(\vec{S}_{1} \cdot \vec{v}_{2}\right)\left(\vec{S}_{2} \cdot \vec{n}\right)\left(\vec{v}_{1} \cdot \vec{n}\right) \\
& -20\left(\vec{S}_{1} \cdot \vec{n}\right)^{2}\left(\vec{S}_{1} \cdot \vec{v}_{2}\right)\left(\vec{S}_{2} \cdot \vec{v}_{1}\right)+20\left(\vec{S}_{1} \cdot \vec{n}\right)^{2}\left(\vec{S}_{1} \cdot \vec{S}_{2}\right)\left(\vec{v}_{1} \cdot \vec{v}_{2}\right) \\
& +10\left(\vec{S}_{1} \cdot \vec{n}\right)\left(\vec{S}_{1} \cdot \vec{v}_{1}\right)\left(\vec{S}_{1} \cdot \vec{S}_{2}\right)\left(\vec{v}_{2} \cdot \vec{n}\right)-10\left(\vec{S}_{1} \cdot \vec{n}\right)\left(\vec{S}_{1} \cdot \vec{v}_{1}\right)\left(\vec{S}_{1} \cdot \vec{v}_{2}\right)\left(\vec{S}_{2} \cdot \vec{n}\right) \\
& +S_{1}^{2}\left[15\left(\vec{S}_{1} \cdot \vec{n}\right)\left(\vec{S}_{2} \cdot \vec{n}\right)\left(\vec{v}_{1} \cdot \vec{v}_{2}\right)-15\left(\vec{S}_{1} \cdot \vec{n}\right)\left(\vec{S}_{2} \cdot \vec{v}_{1}\right)\left(\vec{v}_{2} \cdot \vec{n}\right)\right. \\
& -4\left(\vec{S}_{1} \cdot \vec{S}_{2}\right)\left(\vec{v}_{1} \cdot \vec{v}_{2}\right)+5\left(\vec{S}_{1} \cdot \vec{S}_{2}\right)\left(\vec{v}_{1} \cdot \vec{n}\right)\left(\vec{v}_{2} \cdot \vec{n}\right) \\
& \left.\left.-5\left(\vec{S}_{1} \cdot \vec{v}_{2}\right)\left(\vec{S}_{2} \cdot \vec{n}\right)\left(\vec{v}_{1} \cdot \vec{n}\right)+4\left(\vec{S}_{1} \cdot \vec{v}_{2}\right)\left(\vec{S}_{2} \cdot \vec{v}_{1}\right)\right]\right\}
\end{aligned}
$$

Fig. 2(b4) $=\frac{C_{1\left(\mathrm{BS}^{3}\right)}}{2} \frac{G}{m_{1}^{2} r^{5}}\left[35\left(\vec{S}_{1} \cdot \vec{n}\right)^{3}\left(\vec{S}_{2} \cdot \vec{v}_{2}\right)\left(\vec{v}_{1} \cdot \vec{n}\right)-70\left(\vec{S}_{1} \cdot \vec{n}\right)^{3}\left(\vec{S}_{2} \cdot \vec{v}_{1}\right)\left(\vec{v}_{2} \cdot \vec{n}\right)\right.$

$-70\left(\vec{S}_{1} \cdot \vec{n}\right)^{2}\left(\vec{S}_{1} \cdot \vec{v}_{2}\right)\left(\vec{S}_{2} \cdot \vec{n}\right)\left(\vec{v}_{1} \cdot \vec{n}\right)+35\left(\vec{S}_{1} \cdot \vec{n}\right)^{2}\left(\vec{S}_{1} \cdot \vec{v}_{1}\right)\left(\vec{S}_{2} \cdot \vec{n}\right)\left(\vec{v}_{2} \cdot \vec{n}\right)$

$+10\left(\vec{S}_{1} \cdot \vec{n}\right)\left(\vec{S}_{1} \cdot \vec{v}_{1}\right)\left(\vec{S}_{1} \cdot \vec{v}_{2}\right)\left(\vec{S}_{2} \cdot \vec{n}\right)+35\left(\vec{S}_{1} \cdot \vec{n}\right)^{2}\left(\vec{S}_{1} \cdot \vec{S}_{2}\right)\left(\vec{v}_{1} \cdot \vec{n}\right)\left(\vec{v}_{2} \cdot \vec{n}\right)$

$-15 S_{1}^{2}\left(\vec{S}_{1} \cdot \vec{n}\right)\left(\vec{S}_{2} \cdot \vec{v}_{2}\right)\left(\vec{v}_{1} \cdot \vec{n}\right)+10\left(\vec{S}_{1} \cdot \vec{n}\right)\left(\vec{S}_{1} \cdot \vec{v}_{2}\right)\left(\vec{S}_{1} \cdot \vec{S}_{2}\right)\left(\vec{v}_{1} \cdot \vec{n}\right)$

$+30 S_{1}^{2}\left(\vec{S}_{1} \cdot \vec{n}\right)\left(\vec{S}_{2} \cdot \vec{v}_{1}\right)\left(\vec{v}_{2} \cdot \vec{n}\right)-20\left(\vec{S}_{1} \cdot \vec{n}\right)\left(\vec{S}_{1} \cdot \vec{v}_{1}\right)\left(\vec{S}_{1} \cdot \vec{S}_{2}\right)\left(\vec{v}_{2} \cdot \vec{n}\right)$

$-5\left(\vec{S}_{1} \cdot \vec{n}\right)^{2}\left(\vec{S}_{1} \cdot \vec{S}_{2}\right)\left(\vec{v}_{1} \cdot \vec{v}_{2}\right)-20\left(\vec{S}_{1} \cdot \vec{n}\right)^{2}\left(\vec{S}_{1} \cdot \vec{v}_{1}\right)\left(\vec{S}_{2} \cdot \vec{v}_{2}\right)$

$+40\left(\vec{S}_{1} \cdot \vec{n}\right)^{2}\left(\vec{S}_{1} \cdot \vec{v}_{2}\right)\left(\vec{S}_{2} \cdot \vec{v}_{1}\right)+10 S_{1}^{2}\left(\vec{S}_{1} \cdot \vec{v}_{2}\right)\left(\vec{S}_{2} \cdot \vec{n}\right)\left(\vec{v}_{1} \cdot \vec{n}\right)$

$-5 S_{1}^{2}\left(\vec{S}_{1} \cdot \vec{v}_{1}\right)\left(\vec{S}_{2} \cdot \vec{n}\right)\left(\vec{v}_{2} \cdot \vec{n}\right)-5 S_{1}^{2}\left(\vec{S}_{1} \cdot \vec{S}_{2}\right)\left(\vec{v}_{1} \cdot \vec{n}\right)\left(\vec{v}_{2} \cdot \vec{n}\right)$

$+S_{1}^{2}\left(\vec{S}_{1} \cdot \vec{S}_{2}\right)\left(\vec{v}_{1} \cdot \vec{v}_{2}\right)+4 S_{1}^{2}\left(\vec{S}_{1} \cdot \vec{v}_{1}\right)\left(\vec{S}_{2} \cdot \vec{v}_{2}\right)$

$\left.-8 S_{1}^{2}\left(\vec{S}_{1} \cdot \vec{v}_{2}\right)\left(\vec{S}_{2} \cdot \vec{v}_{1}\right)\right]$

$+\frac{C_{1\left(\mathrm{BS}^{3}\right)}}{2} \frac{G}{m_{1}^{2} r^{4}}\left[5\left(\dot{\vec{S}}_{1} \cdot \vec{S}_{2}\right)\left(\vec{S}_{1} \cdot \vec{n}\right)^{2}\left(\vec{v}_{2} \cdot \vec{n}\right)\right.$

$+8\left(\dot{\vec{S}}_{1} \cdot \vec{S}_{1}\right)\left(\vec{S}_{1} \cdot \vec{n}\right)\left(\vec{S}_{2} \cdot \vec{v}_{2}\right)-2\left(\dot{\vec{S}}_{1} \cdot \vec{S}_{1}\right)\left(\vec{S}_{1} \cdot \vec{v}_{2}\right)\left(\vec{S}_{2} \cdot \vec{n}\right)$

$-10\left(\dot{\vec{S}}_{1} \cdot \vec{S}_{1}\right)\left(\vec{S}_{1} \cdot \vec{n}\right)\left(\vec{S}_{2} \cdot \vec{n}\right)\left(\vec{v}_{2} \cdot \vec{n}\right)-6\left(\dot{\vec{S}}_{1} \cdot \vec{S}_{2}\right)\left(\vec{S}_{1} \cdot \vec{n}\right)\left(\vec{S}_{1} \cdot \vec{v}_{2}\right)$

$+10\left(\dot{\vec{S}}_{1} \cdot \vec{v}_{2}\right)\left(\vec{S}_{1} \cdot \vec{n}\right)^{2}\left(\vec{S}_{2} \cdot \vec{n}\right)-6\left(\dot{\vec{S}}_{1} \cdot \vec{v}_{2}\right)\left(\vec{S}_{1} \cdot \vec{n}\right)\left(\vec{S}_{1} \cdot \vec{S}_{2}\right)$

$+20\left(\dot{\vec{S}}_{1} \cdot \vec{n}\right)\left(\vec{S}_{1} \cdot \vec{v}_{2}\right)\left(\vec{S}_{1} \cdot \vec{n}\right)\left(\vec{S}_{2} \cdot \vec{n}\right)-6\left(\dot{\vec{S}}_{1} \cdot \vec{n}\right)\left(\vec{S}_{1} \cdot \vec{v}_{2}\right)\left(\vec{S}_{1} \cdot \vec{S}_{2}\right)$

$+10\left(\dot{\vec{S}}_{1} \cdot \vec{n}\right)\left(\vec{S}_{1} \cdot \vec{n}\right)\left(\vec{S}_{1} \cdot \vec{S}_{2}\right)\left(\vec{v}_{2} \cdot \vec{n}\right)-15\left(\dot{\vec{S}}_{1} \cdot \vec{n}\right)\left(\vec{S}_{1} \cdot \vec{n}\right)^{2}\left(\vec{S}_{2} \cdot \vec{v}_{2}\right)$

$\left.-5 S_{1}^{2}\left(\dot{\vec{S}}_{1} \cdot \vec{n}\right)\left(\vec{S}_{2} \cdot \vec{n}\right)\left(\vec{v}_{2} \cdot \vec{n}\right)-S_{1}^{2}\left(\dot{\vec{S}}_{1} \cdot \vec{v}_{2}\right)\left(\vec{S}_{2} \cdot \vec{n}\right)+4 S_{1}^{2}\left(\dot{\vec{S}}_{1} \cdot \vec{n}\right)\left(\vec{S}_{2} \cdot \vec{v}_{2}\right)\right]$,

Fig. $2(\mathrm{c} 1)=\frac{C_{1\left(\mathrm{ES}^{4}\right)}}{16} \frac{G m_{2}}{m_{1}^{3} r^{5}}\left\{\left[35\left(\vec{S}_{1} \cdot \vec{n}\right)^{4}-30 S_{1}^{2}\left(\vec{S}_{1} \cdot \vec{n}\right)^{2}+3 S_{1}^{4}\right]\left(2+3 v_{1}^{2}+3 v_{2}^{2}\right)\right.$

$-140\left(\vec{S}_{1} \cdot \vec{n}\right)^{3}\left(\vec{S}_{1} \cdot \vec{v}_{1}\right)\left(\vec{v}_{1} \cdot \vec{n}\right)+60\left(\vec{S}_{1} \cdot \vec{n}\right)^{2}\left(\vec{S}_{1} \cdot \vec{v}_{1}\right)^{2}-12 S_{1}^{2}\left(\vec{S}_{1} \cdot \vec{v}_{1}\right)^{2}$

$\left.+60 S_{1}^{2}\left(\vec{S}_{1} \cdot \vec{n}\right)\left(\vec{S}_{1} \cdot \vec{v}_{1}\right)\left(\vec{v}_{1} \cdot \vec{n}\right)\right\}$

$+\frac{C_{1\left(\mathrm{ES}^{4}\right)}}{8} \frac{G m_{2}}{m_{1}^{3} r^{4}}\left[5 S_{1}^{2}\left(\vec{S}_{1} \cdot \vec{n}\right)^{2}\left(\vec{a}_{1} \cdot \vec{n}\right)-2 S_{1}^{2}\left(\vec{S}_{1} \cdot \vec{n}\right)\left(\vec{S}_{1} \cdot \vec{a}_{1}\right)-S_{1}^{4}\left(\vec{a}_{1} \cdot \vec{n}\right)\right]$ 


$$
\begin{aligned}
& +\frac{\left.C_{1(\mathrm{ES}} 4\right)}{12} \frac{G m_{2}}{m_{1}^{3} r^{3}}\left[3\left(\ddot{\vec{S}}_{1} \cdot \vec{S}_{1}\right)\left(\vec{S}_{1} \cdot \vec{n}\right)^{2}+3 S_{1}^{2}\left(\ddot{\vec{S}}_{1} \cdot \vec{n}\right)\left(\vec{S}_{1} \cdot \vec{n}\right)\right. \\
& -2 S_{1}^{2}\left(\ddot{\vec{S}}_{1} \cdot \vec{S}_{1}\right)+3 S_{1}^{2}\left(\dot{\vec{S}}_{1} \cdot \vec{n}\right)^{2}+3 \dot{S}_{1}^{2}\left(\vec{S}_{1} \cdot \vec{n}\right)^{2} \\
& \left.+12\left(\dot{\vec{S}}_{1} \cdot \vec{n}\right)\left(\dot{\vec{S}}_{1} \cdot \vec{S}_{1}\right)\left(\vec{S}_{1} \cdot \vec{n}\right)-2 \dot{S}_{1}^{2} S_{1}^{2}-4\left(\overrightarrow{\vec{S}}_{1} \cdot \vec{S}_{1}\right)^{2}\right],
\end{aligned}
$$

Fig. $2(\mathrm{c} 2)=-\frac{C_{1\left(\mathrm{ES}^{4}\right)}}{16} \frac{G m_{2}}{m_{1}^{3} r^{5}}\left[315\left(\vec{S}_{1} \cdot \vec{n}\right)^{4}\left(\vec{v}_{1} \cdot \vec{n}\right)\left(\vec{v}_{2} \cdot \vec{n}\right)-210 S_{1}^{2}\left(\vec{S}_{1} \cdot \vec{n}\right)^{2}\left(\vec{v}_{1} \cdot \vec{n}\right)\left(\vec{v}_{2} \cdot \vec{n}\right)\right.$

$$
\begin{aligned}
& +15 S_{1}^{4}\left(\vec{v}_{1} \cdot \vec{n}\right)\left(\vec{v}_{2} \cdot \vec{n}\right)-140\left(\vec{S}_{1} \cdot \vec{n}\right)^{3}\left(\vec{S}_{1} \cdot \vec{v}_{1}\right)\left(\vec{v}_{2} \cdot \vec{n}\right) \\
& +60 S_{1}^{2}\left(\vec{S}_{1} \cdot \vec{v}_{1}\right)\left(\vec{S}_{1} \cdot \vec{n}\right)\left(\vec{v}_{2} \cdot \vec{n}\right)-140\left(\vec{S}_{1} \cdot \vec{n}\right)^{3}\left(\vec{S}_{1} \cdot \vec{v}_{2}\right)\left(\vec{v}_{1} \cdot \vec{n}\right)
\end{aligned}
$$$$
+60 S_{1}^{2}\left(\vec{S}_{1} \cdot \vec{n}\right)\left(\vec{S}_{1} \cdot \vec{v}_{2}\right)\left(\vec{v}_{1} \cdot \vec{n}\right)-35\left(\vec{S}_{1} \cdot \vec{n}\right)^{4}\left(\vec{v}_{1} \cdot \vec{v}_{2}\right)
$$$$
+60\left(\vec{S}_{1} \cdot \vec{n}\right)^{2}\left(\vec{S}_{1} \cdot \vec{v}_{1}\right)\left(\vec{S}_{1} \cdot \vec{v}_{2}\right)-12 S_{1}^{2}\left(\vec{S}_{1} \cdot \vec{v}_{1}\right)\left(\vec{S}_{1} \cdot \vec{v}_{2}\right)
$$$$
\left.+30 S_{1}^{2}\left(\vec{S}_{1} \cdot \vec{n}\right)^{2}\left(\vec{v}_{1} \cdot \vec{v}_{2}\right)-3 S_{1}^{4}\left(\vec{v}_{1} \cdot \vec{v}_{2}\right)\right]
$$$$
-\frac{C_{1\left(\mathrm{ES}^{4}\right)}}{4} \frac{G m_{2}}{m_{1}^{3} r^{4}}\left[5\left(\dot{\vec{S}}_{1} \cdot \vec{v}_{2}\right)\left(\vec{S}_{1} \cdot \vec{n}\right)^{3}+15\left(\dot{\vec{S}}_{1} \cdot \vec{S}_{1}\right)\left(\vec{S}_{1} \cdot \vec{n}\right)^{2}\left(\vec{v}_{2} \cdot \vec{n}\right)\right.
$$$$
-35\left(\dot{\vec{S}}_{1} \cdot \vec{n}\right)\left(\vec{S}_{1} \cdot \vec{n}\right)^{3}\left(\vec{v}_{2} \cdot \vec{n}\right)-3 S_{1}^{2}\left(\dot{\vec{S}}_{1} \cdot \vec{v}_{2}\right)\left(\vec{S}_{1} \cdot \vec{n}\right)-3 S_{1}^{2}\left(\dot{\vec{S}}_{1} \cdot \vec{S}_{1}\right)\left(\vec{v}_{2} \cdot \vec{n}\right)
$$$$
+15 S_{1}^{2}\left(\dot{\vec{S}}_{1} \cdot \vec{n}\right)\left(\vec{S}_{1} \cdot \vec{n}\right)\left(\vec{v}_{2} \cdot \vec{n}\right)-6\left(\dot{\vec{S}}_{1} \cdot \vec{S}_{1}\right)\left(\vec{S}_{1} \cdot \vec{v}_{2}\right)\left(\vec{S}_{1} \cdot \vec{n}\right)
$$$$
\left.+15\left(\dot{\vec{S}}_{1} \cdot \vec{n}\right)\left(\vec{S}_{1} \cdot \vec{n}\right)^{2}\left(\vec{S}_{1} \cdot \vec{v}_{2}\right)-3 S_{1}^{2}\left(\dot{\vec{S}}_{1} \cdot \vec{n}\right)\left(\vec{S}_{1} \cdot \vec{v}_{2}\right)\right] \text {, }
$$

Fig. $2(\mathrm{c} 3)=\frac{C_{1\left(\mathrm{ES}^{4}\right)}}{2} \frac{G m_{2}}{m_{1}^{3} r^{5}}\left(\vec{v}_{1} \cdot \vec{v}_{2}\right)\left[30 S_{1}^{2}\left(\vec{S}_{1} \cdot \vec{n}\right)^{2}-35\left(\vec{S}_{1} \cdot \vec{n}\right)^{4}-3 S_{1}^{4}\right]$

$$
\begin{aligned}
& +\frac{C_{1\left(\mathrm{ES} S^{4}\right)}}{2} \frac{G m_{2}}{m_{1}^{3} r^{4}}\left[5\left(\dot{\vec{S}}_{1} \cdot \vec{v}_{2}\right)\left(\vec{S}_{1} \cdot \vec{n}\right)^{3}+15\left(\dot{\vec{S}}_{1} \cdot \vec{n}\right)\left(n \cdot S_{1}\right)^{2}\left(\vec{S}_{1} \cdot \vec{v}_{2}\right)\right. \\
& \left.-3 S_{1}^{2}\left(\dot{\vec{S}}_{1} \cdot \vec{v}_{2}\right)\left(\vec{S}_{1} \cdot \vec{n}\right)-6\left(\dot{\vec{S}}_{1} \cdot \vec{S}_{1}\right)\left(\vec{S}_{1} \cdot \vec{n}\right)\left(\vec{S}_{1} \cdot \vec{v}_{2}\right)-3 S_{1}^{2}\left(\dot{\vec{S}}_{1} \cdot \vec{n}\right)\left(\vec{S}_{1} \cdot \vec{v}_{2}\right)\right] .
\end{aligned}
$$

Notice that almost all these graphs contain higher-order time derivative terms, notably even at second order, whereas at the LO no higher-order time derivatives appeared yet [28].

\subsection{Two-graviton exchange}

There are 8 graphs of two-graviton exchange in this sector, shown in figure 3 , none of which contains time derivatives. The graph 3 (a1) contains a new two-graviton coupling to the hexadecapole.

The graphs in figure 3 have the following values:

Fig. $3(\mathrm{a} 1)=-\frac{3 C_{1\left(\mathrm{ES}^{4}\right)}}{8} \frac{G^{2} m_{2}^{2}}{m_{1}^{3} r^{6}}\left[95\left(\vec{S}_{1} \cdot \vec{n}\right)^{4}-81 S_{1}^{2}\left(\vec{S}_{1} \cdot \vec{n}\right)^{2}+8 S_{1}^{4}\right]$,

Fig. $3(\mathrm{a} 2)=-\frac{C_{1\left(\mathrm{ES}^{4}\right)}}{8} \frac{G^{2} m_{2}}{m_{1}^{2} r^{6}}\left[3 S_{1}^{4}-30 S_{1}^{2}\left(\vec{S}_{1} \cdot \vec{n}\right)^{2}+35\left(\vec{S}_{1} \cdot \vec{n}\right)^{4}\right]$,

Fig. 3(b1) $=-\frac{C_{1\left(\mathrm{BS}^{3}\right)}}{3} \frac{G^{2} m_{2}}{m_{1}^{2} r^{6}}\left[441\left(\vec{S}_{1} \cdot \vec{n}\right)^{3}\left(\vec{S}_{2} \cdot \vec{n}\right)-189 S_{1}^{2}\left(\vec{S}_{1} \cdot \vec{n}\right)\left(\vec{S}_{2} \cdot \vec{n}\right)\right.$

$$
\left.-183\left(\vec{S}_{1} \cdot \vec{S}_{2}\right)\left(\vec{S}_{1} \cdot \vec{n}\right)^{2}+35 S_{1}^{2}\left(\vec{S}_{1} \cdot \vec{S}_{2}\right)\right]
$$




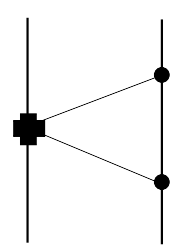

(a1)

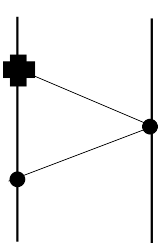

(a2)

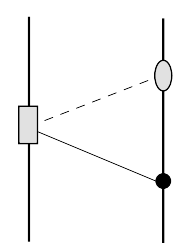

(b1)

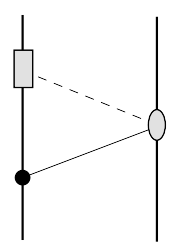

(b2)

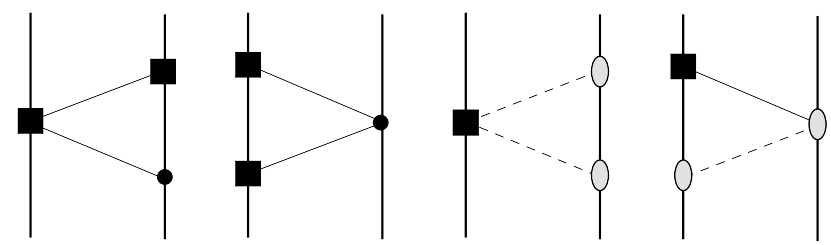

(c1)

(c2)

(d1)

(d2)

Figure 3. The Feynman graphs of two-graviton exchange at the NLO quartic-in-spin interaction. These graphs contain all relevant interactions among the mass, spin and spin-induced multipoles up to the hexadecapole. In particular at this nonlinear level there are also interactions with the various multipoles on two different points of the worldline, which occurs as of the NLO spinsquared sector $[4,13,14]$, such as a spin dipole and a spin-induced quadrupole or two spin-induced quadrupoles on the same worldline. The graph (a1) contains a new two-graviton coupling to the hexadecapole.

Fig. $3(\mathrm{~b} 2)=-2 C_{1\left(\mathrm{BS}^{3}\right)} \frac{G^{2}}{m_{1} r^{6}}\left[3 S_{1}^{2}\left(\vec{S}_{1} \cdot \vec{S}_{2}\right)-15 S_{1}^{2}\left(\vec{S}_{1} \cdot \vec{n}\right)\left(\vec{S}_{2} \cdot \vec{n}\right)-15\left(\vec{S}_{1} \cdot \vec{S}_{2}\right)\left(\vec{S}_{1} \cdot \vec{n}\right)^{2}\right.$

$$
\left.+35\left(\vec{S}_{1} \cdot \vec{n}\right)^{3}\left(\vec{S}_{2} \cdot \vec{n}\right)\right]
$$

Fig. $3(\mathrm{c} 1)=-\frac{9 C_{1\left(\mathrm{ES}^{2}\right)} C_{2\left(\mathrm{ES}^{2}\right)}}{2} \frac{G^{2}}{m_{1} r^{6}}\left[S_{1}^{2} S_{2}^{2}-4 S_{1}^{2}\left(\vec{S}_{2} \cdot \vec{n}\right)-4 S_{2}^{2}\left(\vec{S}_{1} \cdot \vec{n}\right)^{2}+\left(\vec{S}_{1} \cdot \vec{S}_{2}\right)^{2}\right.$

$$
\left.-12\left(\vec{S}_{1} \cdot \vec{S}_{2}\right)\left(\vec{S}_{1} \cdot \vec{n}\right)\left(\vec{S}_{2} \cdot \vec{n}\right)+24\left(\vec{S}_{1} \cdot \vec{n}\right)^{2}\left(\vec{S}_{2} \cdot \vec{n}\right)^{2}\right],
$$

Fig. $3(\mathrm{c} 2)=-\frac{C_{1\left(\mathrm{ES}^{2}\right)}^{2}}{8} \frac{G^{2} m_{2}}{m_{1}^{2} r^{6}}\left[3\left(\vec{S}_{1} \cdot \vec{n}\right)^{2}-S_{1}^{2}\right]^{2}$,

Fig. $3(\mathrm{~d} 1)=-\frac{C_{1\left(\mathrm{ES}^{2}\right)}}{2} \frac{G^{2}}{m_{1} r^{6}}\left[S_{1}^{2} S_{2}^{2}+\left(\vec{S}_{1} \cdot \vec{S}_{2}\right)^{2}+3 S_{1}^{2}\left(\vec{S}_{2} \cdot \vec{n}\right)^{2}+9\left(\vec{S}_{1} \cdot \vec{n}\right)^{2}\left(\vec{S}_{2} \cdot \vec{n}\right)^{2}\right.$

$$
\left.-6\left(\vec{S}_{1} \cdot \vec{n}\right)\left(\vec{S}_{1} \cdot \vec{S}_{2}\right)\left(\vec{S}_{2} \cdot \vec{n}\right)\right]
$$

Fig. $3(\mathrm{~d} 2)=-2 C_{1\left(\mathrm{ES}^{2}\right)} \frac{G^{2}}{m_{1} r^{6}}\left[3\left(\vec{S}_{1} \cdot \vec{n}\right)^{2}-S_{1}^{2}\right]\left[3\left(\vec{S}_{1} \cdot \vec{n}\right)\left(\vec{S}_{2} \cdot \vec{n}\right)-\left(\vec{S}_{1} \cdot \vec{S}_{2}\right)\right]$.

\subsection{Cubic self-interaction}

There are 5 graphs of cubic self-interaction in this sector, shown in figure 4, none of which contains time-dependent self-interaction, similar to the NLO quadratic-in-spin sector of even parity [14]. These graphs contain all possible interactions among the mass, spin and spin-induced multipoles up to the hexadecapole, similar to the nonlinear graphs of two-graviton exchange. 


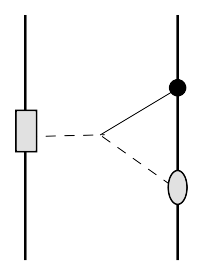

(a1)

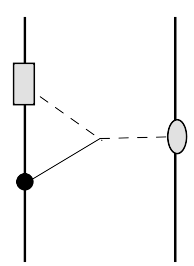

(a2)

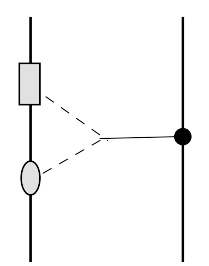

(a3)

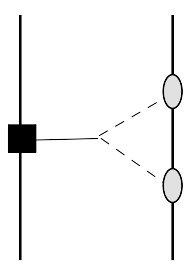

(b1)

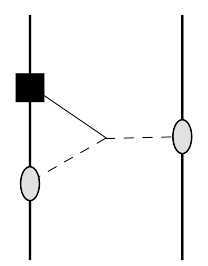

(b2)

Figure 4. The Feynman graphs with cubic self-gravitational interaction, i.e. at one-loop level, at the NLO quartic-in-spin interaction. There are no vertices with time dependence here, similar to the NLO quadratic-in-spin sector of even-parity [14]. These graphs contain all possible interactions among the mass, spin and spin-induced multipoles up to the hexadecapole.

The graphs in figure 4 have the following values:

Fig. $4(\mathrm{a} 1)=-\frac{4 C_{1\left(\mathrm{BS}^{3}\right)}}{3} \frac{G^{2} m_{2}}{m_{1}^{2} r^{6}}\left[9 S_{1}^{2}\left(\vec{S}_{1} \cdot \vec{n}\right)\left(\vec{S}_{2} \cdot \vec{n}\right)-24\left(\vec{S}_{1} \cdot \vec{n}\right)^{3}\left(\vec{S}_{2} \cdot \vec{n}\right)\right.$

$$
\left.+12\left(\vec{S}_{1} \cdot \vec{n}\right)^{2}\left(\vec{S}_{1} \cdot \vec{S}_{2}\right)-2 S_{1}^{2}\left(\vec{S}_{1} \cdot \vec{S}_{2}\right)\right]
$$

Fig. $4(\mathrm{a} 2)=-3 C_{1\left(\mathrm{BS}^{3}\right)} \frac{G^{2}}{m_{1} r^{6}}\left[4 S_{1}^{2}\left(\vec{S}_{1} \cdot \vec{n}\right)\left(\vec{S}_{2} \cdot \vec{n}\right)-10\left(\vec{S}_{1} \cdot \vec{n}\right)^{3}\left(\vec{S}_{2} \cdot \vec{n}\right)\right.$

$$
\left.+5\left(\vec{S}_{1} \cdot \vec{n}\right)^{2}\left(\vec{S}_{1} \cdot \vec{S}_{2}\right)-S_{1}^{2}\left(\vec{S}_{1} \cdot \vec{S}_{2}\right)\right]
$$

Fig. $4(\mathrm{a} 3)=C_{1\left(\mathrm{BS}^{3}\right)} \frac{G^{2} m_{2}}{m_{1}^{2} r^{6}}\left(\vec{S}_{1} \cdot \vec{n}\right)^{2}\left[3 S_{1}^{2}-5\left(\vec{S}_{1} \cdot \vec{n}\right)^{2}\right]$,

Fig. $4(\mathrm{~b} 1)=-C_{1\left(\mathrm{ES}^{2}\right)} \frac{G^{2}}{m_{1} r^{6}}\left[24\left(\vec{S}_{1} \cdot \vec{n}\right)^{2}\left(\vec{S}_{2} \cdot \vec{n}\right)^{2}-6 S_{1}^{2}\left(\vec{S}_{2} \cdot \vec{n}\right)^{2}+\left(\vec{S}_{1} \cdot \vec{S}_{2}\right)^{2}-S_{1}^{2} S_{2}^{2}\right.$

$$
\left.-12\left(\vec{S}_{1} \cdot \vec{n}\right)\left(\vec{S}_{1} \cdot \vec{S}_{2}\right)\left(\vec{S}_{2} \cdot \vec{n}\right)\right] \text {, }
$$

Fig. $4(\mathrm{~b} 2)=-C_{1\left(\mathrm{ES}^{2}\right)} \frac{G^{2}}{m_{1} r^{6}}\left[6\left(\vec{S}_{1} \cdot \vec{n}\right)^{3}\left(\vec{S}_{2} \cdot \vec{n}\right)-3\left(\vec{S}_{1} \cdot \vec{n}\right)^{2}\left(\vec{S}_{1} \cdot \vec{S}_{2}\right)-S_{1}^{2}\left(\vec{S}_{1} \cdot \vec{S}_{2}\right)\right]$.

\section{Composite worldline couplings}

The formulation of the EFT of a spinning particle in [14] assumed an initial covariant gauge of the rotational DOFs in terms of the linear momentum, as originally put forward by Tulczyjew [50] (extended by Dixon [51] later to higher-multipoles), which was proven to be uniquely distinguished $[52,53]$. As detailed in section 4 of [4], and pointed out already in [14], this gives rise to composite worldline couplings in sectors of higher-spin as of the NLO cubic-in-spin sector as the linear momentum can no longer be considered independent of the spin

$$
p_{\mu}=-\frac{\partial L}{\partial u^{\mu}}=m \frac{u_{\mu}}{\sqrt{u}^{2}}+\mathcal{O}\left(R S^{2}\right) .
$$

The correction to the linear momentum which was already required for the NLO cubic-inspin sector [4] is given by

$$
\Delta p_{\kappa}\left[S^{2}\right] \equiv p_{\kappa}\left[S^{2}\right]-\bar{p}_{\kappa} \simeq \frac{C_{\mathrm{ES}^{2}}}{2 m} S^{\mu} S^{\nu}\left(\frac{2}{u} R_{\mu \alpha \nu \kappa} u^{\alpha}-\frac{1}{u^{3}} R_{\mu \alpha \nu \beta} u^{\alpha} u^{\beta} u_{\kappa}\right),
$$


where $\bar{p}_{\kappa} \equiv \frac{m}{u} u_{\kappa}$ is the leading approximation to the linear momentum. At this order it is clear from using eq. (4.8) of [14] that the spin-induced multipole itself, rewritten as a contraction of spin tensors, has no $u^{\mu}$ dependence and thus no additional contribution to the linear momentum.

At the current NLO quartic-in-spin sector we also have to consider the next spindependent correction to the linear momentum. Similar to the correction in eq. (4.2), by first treating the product of spin vectors as independent of $u^{\mu}$, one gets a contribution

$$
\begin{aligned}
\Delta p_{\kappa}\left[S^{3}\right] \equiv p_{\kappa}\left[S^{3}\right]-p_{\kappa}\left[S^{2}\right] & \\
\simeq \frac{C_{B S^{3}}}{12 m^{2}} S^{\mu} S^{\nu} S^{\lambda}\left[\frac { 1 } { u } \left(\epsilon_{\alpha \beta \kappa \mu} D_{\lambda} R_{\delta \nu}^{\alpha \beta} u^{\delta}\right.\right. & \left.+\epsilon_{\alpha \beta \gamma \mu} D_{\lambda} R_{\kappa \nu}^{\alpha \beta} u^{\gamma}\right) \\
& \left.\quad-\frac{1}{u^{3}} \epsilon_{\alpha \beta \gamma \mu} D_{\lambda} R^{\alpha \beta}{ }_{\delta \nu} u^{\gamma} u^{\delta} u_{\kappa}\right] .
\end{aligned}
$$

Yet here with a tensor product of 3 spin vectors, after trading 2 spin vectors by a contraction of 2 spin tensors (using again eq. (4.8) of [14]), which is independent of $u^{\mu}$, the dependence in $u^{\mu}$ of a third spin vector in the product still has to be considered. This dependence in fact gives rise to an additional contribution to the correction in eq. (4.3)

$$
\Delta p_{\kappa}^{\text {extra }}\left[S^{3}\right] \simeq \frac{C_{B S^{3}}}{6 m^{2}} \frac{D_{\lambda} B_{\mu \nu}}{u^{2}} S^{\mu} S^{\nu}\left[* S_{\kappa}^{\lambda}-S^{\lambda} \frac{u_{\kappa}}{u}\right],
$$

where we use here the dual spin tensor defined by,

$$
* S_{\alpha \beta} \equiv \frac{1}{2} \epsilon_{\alpha \beta \mu \nu} S^{\mu \nu}
$$

as introduced in [14]. However, as it turns out the additional correction in eq. (4.4) will not contribute to the present sector.

These corrections should be implemented first in the minimal coupling part of the effective action of a spinning particle, which is recast in the form [14]:

$$
L_{S}=-\frac{1}{2} \hat{S}_{a b} \hat{\Omega}_{\text {flat }}^{a b}-\frac{1}{2} \hat{S}_{a b} \omega_{\mu}^{a b} u^{\mu}-\frac{\hat{S}_{a b} p^{b}}{p^{2}} \frac{D p^{a}}{D \sigma},
$$

with lowercase Latin indices for the locally flat frame, and where the Ricci rotation coefficients, $\omega_{\mu}^{a b}$, are used. The new couplings arise from substituting in the linear momentum in the canonical gauge into the linear-in-spin couplings, and into the extra term that appears last in eq. (4.6), which as noted in section 2 stands for the Thomas precession and was related in [14] to the gauge of the rotational DOFs.

It is important to stress that the issue here is not about going from a covariant gauge to a "non-covariant" gauge, rather it is about going from the spin-independent approximation of the linear momentum to its spin-dependent completion.

From eq. (4.6) we obtain the following terms that yield new higher-order in spin couplings [4, 14]:

$$
L_{S \rightarrow S^{3}, S^{4}}=\omega_{\mu}^{i j} u^{\mu} \frac{\hat{S}_{i k} p^{k} p_{j}}{p\left(p+p^{0}\right)}-\omega_{\mu}^{0 i} u^{\mu} \frac{\hat{S}_{i j} p^{j}}{p}+\frac{\hat{S}_{i j} p^{i} \dot{p}^{j}}{p\left(p+p^{0}\right)},
$$




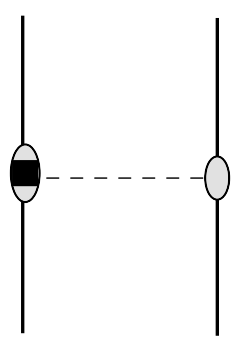

(a1)

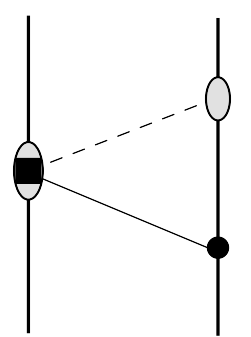

(b1)

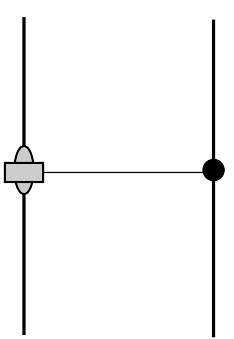

(a2)

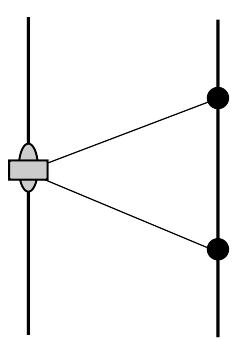

(b2)

Figure 5. The Feynman graphs of one- and two-graviton exchange from composite worldline couplings that appear at the NLO quartic-in-spin interaction. These graphs with the composite couplings, that are cubic and quartic in the spin, yield similar graphs to the corresponding ones with the elementary spin-induced octupole and hexadecapole in figure 2(b1), (c1), and figure 3(a1), (b1).

where all the indices are in the locally flat frame, and the canonical gauge is applied. The first two terms in eq. (4.7) yield new two-graviton couplings, and the last term yields new one-graviton couplings with higher-order time derivatives. Plugging in the corrections to the linear momentum from eqs. (4.2), (4.3) in eq. (4.7) to linear order yields new worldlinegraviton couplings that are cubic and quartic in the spin, respectively. As noted above, plugging in eq. (4.7) the additional correction in eq. (4.4) is found not to contribute to the present sector.

As for the new couplings that are cubic in the spin, these can be found in [4]. The new couplings that are quartic in the spin have the following Feynman rules

$$
\hat{\natural}=\int d t-\frac{C_{\left(\mathrm{BS}^{3}\right)}}{6 m^{3}}\left[S_{i} S_{j} \phi_{, i j k}\left(2\left(S^{2} a^{k}-\vec{S} \cdot \vec{a} S_{k}\right)+\dot{\vec{S}} \cdot \vec{S} v^{k}-\vec{S} \cdot \vec{v} \dot{S}_{k}\right)\right],
$$

for the one-graviton coupling, and for a two-graviton coupling

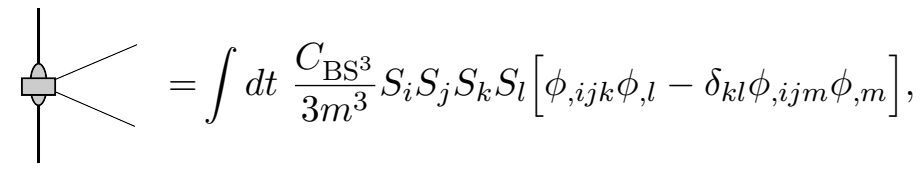

where a gray rectangle mounted on an oval gray blob represents this new type of "composite" quartic-in-spin worldline couplings. Further KK field couplings of this type enter beyond the 5PN order. The Wilson coefficients in these new rules for quartic-in-spin couplings indicate that these cannot be identified with the elementary hexadecapole couplings.

The composite worldline couplings from [4] and the new quartic-in-spin couplings above give rise to 4 additional graphs in this sector, as shown in figure 5, which are similar to those in figure 2 (b1), (c1) and in figure 3 (a1), (b1). The graphs in figure 5 have the following values:

$$
\begin{aligned}
\text { Fig. 5(a1) }= & \frac{3 C_{1\left(\mathrm{ES}^{2}\right)}}{2 m_{1}^{2}} \frac{G}{r^{4}}\left[\left[2 S_{1}^{2}\left(\vec{S}_{2} \cdot \vec{a}_{1}\right)+\left(\dot{\vec{S}}_{1} \cdot \vec{S}_{1}\right)\left(\vec{S}_{2} \cdot \vec{v}_{1}\right)-\left(\dot{\vec{S}}_{1} \cdot \vec{S}_{2}\right)\left(\vec{S}_{1} \cdot \vec{v}_{1}\right)\right]\left(\vec{S}_{1} \cdot \vec{n}\right)\right. \\
& +\left[2 S_{1}^{2}\left(\vec{a}_{1} \cdot \vec{n}\right)+\left(\dot{\vec{S}}_{1} \cdot \vec{S}_{1}\right)\left(\vec{v}_{1} \cdot \vec{n}\right)-\left(\dot{\vec{S}}_{1} \cdot \vec{n}\right)\left(\vec{S}_{1} \cdot \vec{v}_{1}\right)\right]
\end{aligned}
$$




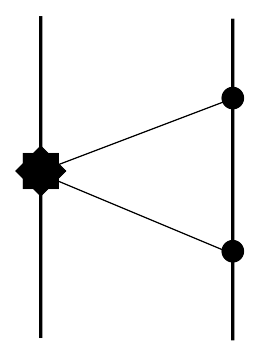

Figure 6. The Feynman graph of two-graviton exchange at the NLO quartic-in-spin interaction, which originates from the new quadratic-in-curvature operator with the hexadecapole and with a new Wilson coefficient.

$$
\begin{aligned}
& \times\left[\vec{S}_{1} \cdot \vec{S}_{2}-5\left(\vec{S}_{1} \cdot \vec{n}\right)\left(\vec{S}_{2} \cdot \vec{n}\right)\right] \\
& \left.-2\left(\vec{S}_{1} \cdot \vec{n}\right)\left(\vec{S}_{1} \cdot \vec{a}_{1}\right)\left(2\left(\vec{S}_{1} \cdot \vec{S}_{2}\right)-5\left(\vec{S}_{1} \cdot \vec{n}\right)\left(\vec{S}_{2} \cdot \vec{n}\right)\right)\right],
\end{aligned}
$$

Fig. $5(\mathrm{~b} 1)=3 C_{1\left(\mathrm{ES}^{2}\right)} \frac{m_{2}}{m_{1}^{2}} \frac{G^{2}}{r^{6}}\left[S_{1}^{2}\left[\vec{S}_{1} \cdot \vec{S}_{2}-4\left(\vec{S}_{1} \cdot \vec{n}\right)\left(\vec{S}_{2} \cdot \vec{n}\right)\right]\right.$

$$
\left.-\left(\vec{S}_{1} \cdot \vec{n}\right)^{2}\left[2\left(\vec{S}_{1} \cdot \vec{S}_{2}\right)-5\left(\vec{S}_{1} \cdot \vec{n}\right)\left(\vec{S}_{2} \cdot \vec{n}\right)\right]\right]
$$

Fig. $5(\mathrm{a} 2)=\frac{1}{2} C_{1\left(\mathrm{BS}^{3}\right)} \frac{m_{2}}{m_{1}^{3}} \frac{G}{r^{4}}\left[S_{1}^{2}-5\left(\vec{S}_{1} \cdot \vec{n}\right)^{2}\right]$

$$
\times\left[2 S_{1}^{2}\left(\vec{a}_{1} \cdot \vec{n}\right)-2\left(\vec{S}_{1} \cdot \vec{n}\right)\left(\vec{S}_{1} \cdot \vec{a}_{1}\right)+\left(\dot{\vec{S}}_{1} \cdot \vec{S}_{1}\right)\left(\vec{v}_{1} \cdot \vec{n}\right)-\left(\dot{\vec{S}}_{1} \cdot \vec{n}\right)\left(\vec{S}_{1} \cdot \vec{v}_{1}\right)\right],
$$

Fig. $5(\mathrm{~b} 2)=C_{1\left(\mathrm{BS}^{3}\right)} \frac{m_{2}^{2}}{m_{1}^{3}} \frac{G^{2}}{r^{6}}\left[S_{1}^{4}-6 S_{1}^{2}\left(\vec{S}_{1} \cdot \vec{n}\right)^{2}+5\left(\vec{S}_{1} \cdot \vec{n}\right)^{4}\right]$.

\section{Quadratic-in-curvature worldline couplings}

In this sector yet a new type of composite coupling, which is quartic in the spin and quadratic in the curvature, should be considered: this would arise from plugging the correction in eq. (4.2) back into the leading non-minimal coupling $L_{\mathrm{ES}^{2}}$ in eq. (2.2), such that $L_{S^{2} \rightarrow S^{4}}$, and the resulting coupling would be preceded by the coefficient $\left(C_{\mathrm{ES}^{2}}\right)^{2}$. However, this coupling turns out to be power-counted beyond the $5 \mathrm{PN}$ order, and is thus not relevant here.

We are left then with the elementary coupling of the hexadecapole to the quadratic electric operator from eq. (2.11) as the single contribution to this sector that is quadratic in the curvature. The Feynman rule in eq. (2.11) gives rise to a single two-graviton exchange graph, shown in figure 6 , the value of which is given by

$$
\text { Fig. } 6=\frac{C_{1\left(\mathrm{E}^{2} \mathrm{~S}^{4}\right)} m_{2}^{2}}{24 m_{1}^{3}} \frac{G^{2}}{r^{6}}\left[S_{1}^{4}-6 S_{1}^{2}\left(\vec{S}_{1} \cdot \vec{n}\right)^{2}+9\left(\vec{S}_{1} \cdot \vec{n}\right)^{4}\right] .
$$

Notice that this introduces a new Wilson coefficient that appears first in this sector. 


\section{Next-to-leading gravitational quartic-in-spin action}

Putting together the $28=23+4+1=12+11+5$ graph values from sections 3$),(4$, and 5 , where the summation also takes into account the exchange of particle labels, $1 \leftrightarrow 2$, we get the final effective action for the complete quartic-in-spin sector

$$
L_{\mathrm{S}^{4}}^{\mathrm{NLO}}=L_{\mathrm{S}_{1}^{2} \mathrm{~S}_{2}^{2}}^{\mathrm{NLO}}+L_{\mathrm{S}_{1}^{3} \mathrm{~S}_{2}}^{\mathrm{NLO}}+L_{\mathrm{S}_{1}^{4}}^{\mathrm{NLO}}+(1 \leftrightarrow 2),
$$

where we have

$$
\begin{aligned}
L_{\mathrm{S}_{1}^{2} \mathrm{~S}_{2}^{2}}^{\mathrm{NLO}}= & \frac{1}{2} C_{1\left(\mathrm{ES}^{2}\right)} C_{2\left(\mathrm{ES}^{2}\right)} \frac{G}{m_{1} m_{2}}\left(\frac{3 L_{(1)}}{8 r^{5}}+\frac{3 L_{(2)}}{4 r^{4}}+\frac{L_{(3)}}{2 r^{3}}\right) \\
& -\frac{9}{2} C_{1\left(\mathrm{ES}^{2}\right)} C_{2\left(\mathrm{ES}^{2}\right)} \frac{G^{2}}{m_{1} r^{6}} L_{(4)}+\frac{1}{2} C_{1\left(\mathrm{ES}^{2}\right)} \frac{G^{2}}{m_{1} r^{6}} L_{(5)},
\end{aligned}
$$

with the following pieces:

$$
\begin{aligned}
& L_{(1)}=+10\left(\vec{S}_{1} \cdot \vec{v}_{1}\right)\left[\vec{S}_{1} \cdot\left(\vec{v}_{1}-\vec{v}_{2}\right)\right]\left(\vec{S}_{2} \cdot \vec{n}\right)^{2}-10\left(\vec{S}_{2} \cdot \vec{v}_{2}\right)\left[\vec{S}_{2} \cdot\left(\vec{v}_{1}-\vec{v}_{2}\right)\right]\left(\vec{S}_{1} \cdot \vec{n}\right)^{2} \\
& -4\left[\vec{S}_{1} \cdot\left(\vec{v}_{1}-\vec{v}_{2}\right)\right]\left[\vec{S}_{2} \cdot\left(\vec{v}_{1}-\vec{v}_{2}\right)\right]\left[\left(\vec{S}_{1} \cdot \vec{S}_{2}\right)-5\left(\vec{S}_{1} \cdot \vec{n}\right)\left(\vec{S}_{2} \cdot \vec{n}\right)\right] \\
& +4 S_{1}^{2}\left(\vec{S}_{2} \cdot \vec{v}_{1}\right)^{2}-2 S_{1}^{2}\left[\vec{S}_{2} \cdot\left(\vec{v}_{1}+\vec{v}_{2}\right)\right]\left(\vec{S}_{2} \cdot \vec{v}_{2}\right) \\
& +4\left(\vec{S}_{1} \cdot \vec{v}_{2}\right)^{2} S_{2}^{2}-2\left[\vec{S}_{1} \cdot\left(\vec{v}_{1}+\vec{v}_{2}\right)\right]\left(\vec{S}_{1} \cdot \vec{v}_{1}\right) S_{2}^{2} \\
& +S_{1}^{2} S_{2}^{2}\left[5 v_{1}^{2}+5 v_{2}^{2}-11\left(\vec{v}_{1} \cdot \vec{v}_{2}\right)-10\left(\vec{v}_{1} \cdot \vec{n}\right)^{2}-10\left(\vec{v}_{2} \cdot \vec{n}\right)^{2}+15\left(\vec{v}_{1} \cdot \vec{n}\right)\left(\vec{v}_{2} \cdot \vec{n}\right)\right] \\
& +10 S_{1}^{2}\left(\vec{S}_{2} \cdot \vec{v}_{2}\right)\left(\vec{S}_{2} \cdot \vec{n}\right)\left[\left(\vec{v}_{1}+\vec{v}_{2}\right) \cdot \vec{n}\right]-10 S_{1}^{2}\left(\vec{S}_{2} \cdot \vec{v}_{1}\right)\left(\vec{S}_{2} \cdot \vec{n}\right)\left[4\left(\vec{v}_{1} \cdot \vec{n}\right)-\left(\vec{v}_{2} \cdot \vec{n}\right)\right] \\
& +10\left(\vec{S}_{1} \cdot \vec{v}_{1}\right)\left(\vec{S}_{1} \cdot \vec{n}\right) S_{2}^{2}\left[\left(\vec{v}_{1}+\vec{v}_{2}\right) \cdot \vec{n}\right]-10 S_{2}^{2}\left(\vec{S}_{1} \cdot \vec{v}_{2}\right)\left(\vec{S}_{1} \cdot \vec{n}\right)\left[4\left(\vec{v}_{2} \cdot \vec{n}\right)-\left(\vec{v}_{1} \cdot \vec{n}\right)\right] \\
& -5\left(\vec{S}_{1} \cdot \vec{n}\right)^{2} S_{2}^{2}\left[3 v_{1}^{2}+5 v_{2}^{2}-14\left(\vec{v}_{2} \cdot \vec{n}\right)^{2}-9\left(\vec{v}_{1} \cdot \vec{v}_{2}\right)+7\left(\vec{v}_{1} \cdot \vec{n}\right)\left(\vec{v}_{2} \cdot \vec{n}\right)\right] \\
& -5 S_{1}^{2}\left(\vec{S}_{2} \cdot \vec{n}\right)^{2}\left[5 v_{1}^{2}+3 v_{2}^{2}-14\left(\vec{v}_{1} \cdot \vec{n}\right)^{2}-9\left(\vec{v}_{1} \cdot \vec{v}_{2}\right)+7\left(\vec{v}_{1} \cdot \vec{n}\right)\left(\vec{v}_{2} \cdot \vec{n}\right)\right] \\
& +2\left(\vec{S}_{1} \cdot \vec{S}_{2}\right)^{2}\left[3 v_{1}^{2}+3 v_{2}^{2}-7\left(\vec{v}_{1} \cdot \vec{v}_{2}\right)-5\left(\vec{v}_{1} \cdot \vec{n}\right)\left(\vec{v}_{2} \cdot \vec{n}\right)\right] \\
& +20\left(\vec{S}_{1} \cdot \vec{S}_{2}\right)\left(\vec{S}_{1} \cdot \vec{v}_{1}\right)\left(\vec{S}_{2} \cdot \vec{n}\right)\left[\left(\vec{v}_{1}-\vec{v}_{2}\right) \cdot \vec{n}\right]-20\left(\vec{S}_{1} \cdot \vec{S}_{2}\right)\left(\vec{S}_{1} \cdot \vec{v}_{2}\right)\left(\vec{S}_{2} \cdot \vec{n}\right)\left(\vec{v}_{1} \cdot \vec{n}\right) \\
& -20\left(\vec{S}_{1} \cdot \vec{S}_{2}\right)\left(\vec{S}_{1} \cdot \vec{n}\right)\left(\vec{S}_{2} \cdot \vec{v}_{2}\right)\left[\left(\vec{v}_{1}-\vec{v}_{2}\right) \cdot \vec{n}\right]-20\left(\vec{S}_{1} \cdot \vec{S}_{2}\right)\left(\vec{S}_{1} \cdot \vec{n}\right)\left(\vec{S}_{2} \cdot \vec{v}_{1}\right)\left(\vec{v}_{2} \cdot \vec{n}\right) \\
& -20\left(\vec{S}_{1} \cdot \vec{S}_{2}\right)\left(\vec{S}_{1} \cdot \vec{n}\right)\left(\vec{S}_{2} \cdot \vec{n}\right)\left[3 v_{1}^{2}+3 v_{2}^{2}-7\left(\vec{v}_{1} \cdot \vec{v}_{2}\right)-7\left(\vec{v}_{1} \cdot \vec{n}\right)\left(\vec{v}_{2} \cdot \vec{n}\right)\right] \\
& -70\left(\vec{S}_{1} \cdot \vec{v}_{1}\right)\left(\vec{S}_{1} \cdot \vec{n}\right)\left(\vec{S}_{2} \cdot \vec{n}\right)^{2}\left[\left(\vec{v}_{1}-\vec{v}_{2}\right) \cdot \vec{n}\right]+70\left(\vec{S}_{1} \cdot \vec{v}_{2}\right)\left(\vec{S}_{1} \cdot \vec{n}\right)\left(\vec{S}_{2} \cdot \vec{n}\right)^{2}\left(\vec{v}_{1} \cdot \vec{n}\right) \\
& +70\left(\vec{S}_{1} \cdot \vec{n}\right)^{2}\left(\vec{S}_{2} \cdot \vec{v}_{2}\right)\left(\vec{S}_{2} \cdot \vec{n}\right)\left[\left(\vec{v}_{1}-\vec{v}_{2}\right) \cdot \vec{n}\right]+70\left(\vec{S}_{1} \cdot \vec{n}\right)^{2}\left(\vec{S}_{2} \cdot \vec{v}_{1}\right)\left(\vec{S}_{2} \cdot \vec{n}\right)\left(\vec{v}_{2} \cdot \vec{n}\right) \\
& +35\left(\vec{S}_{1} \cdot \vec{n}\right)^{2}\left(\vec{S}_{2} \cdot \vec{n}\right)^{2}\left[3 v_{1}^{2}+3 v_{2}^{2}-7\left(\vec{v}_{1} \cdot \vec{v}_{2}\right)-9\left(\vec{v}_{1} \cdot \vec{n}\right)\left(\vec{v}_{2} \cdot \vec{n}\right)\right], \\
& L_{(2)}=-2\left(\vec{S}_{1} \cdot \vec{S}_{2}\right)\left(\vec{S}_{1} \cdot \vec{n}\right)\left[2\left(\vec{S}_{2} \cdot \vec{a}_{2}\right)+\dot{\vec{S}}_{2} \cdot\left(2 \vec{v}_{2}-3 \vec{v}_{1}\right)-5\left(\dot{\vec{S}}_{2} \cdot \vec{n}\right)\left(\vec{v}_{1} \cdot \vec{n}\right)\right] \\
& +2\left(\vec{S}_{1} \cdot \vec{S}_{2}\right)\left(\vec{S}_{2} \cdot \vec{n}\right)\left[2\left(\vec{S}_{1} \cdot \vec{a}_{1}\right)+\dot{\vec{S}}_{1} \cdot\left(2 \vec{v}_{1}-3 \vec{v}_{2}\right)-5\left(\dot{\vec{S}}_{1} \cdot \vec{n}\right)\left(\vec{v}_{2} \cdot \vec{n}\right)\right] \\
& -2\left(\vec{S}_{1} \cdot \vec{S}_{2}\right)\left[\left(\vec{S}_{1} \cdot \dot{\vec{S}}_{2}\right)\left(\vec{v}_{1} \cdot \vec{n}\right)-\left(\dot{\vec{S}}_{1} \cdot \vec{S}_{2}\right)\left(\vec{v}_{2} \cdot \vec{n}\right)\right. \\
& \left.+\left(\vec{S}_{1} \cdot \vec{v}_{1}\right)\left(\dot{\vec{S}}_{2} \cdot \vec{n}\right)-\left(\vec{S}_{2} \cdot \vec{v}_{2}\right)\left(\dot{\vec{S}}_{1} \cdot \vec{n}\right)\right] \\
& +2\left(\dot{\vec{S}}_{1} \cdot \vec{S}_{2}\right)\left(\vec{S}_{1} \cdot \vec{n}\right)\left(\vec{S}_{2} \cdot \vec{v}_{2}\right)-2\left(\vec{S}_{1} \cdot \dot{\vec{S}}_{2}\right)\left(\vec{S}_{2} \cdot \vec{n}\right)\left(\vec{S}_{1} \cdot \vec{v}_{1}\right) \\
& -10\left(\dot{\vec{S}}_{1} \cdot \vec{S}_{2}\right)\left(\vec{S}_{1} \cdot \vec{n}\right)\left(\vec{S}_{2} \cdot \vec{n}\right)\left(\vec{v}_{2} \cdot \vec{n}\right)+10\left(\vec{S}_{1} \cdot \dot{\vec{S}}_{2}\right)\left(\vec{S}_{1} \cdot \vec{n}\right)\left(\vec{S}_{2} \cdot \vec{n}\right)\left(\vec{v}_{1} \cdot \vec{n}\right)
\end{aligned}
$$




$$
\begin{aligned}
& -2\left(\vec{S}_{1} \cdot \dot{\vec{S}}_{2}\right)\left(\vec{S}_{1} \cdot \vec{n}\right)\left[\vec{S}_{2} \cdot\left(2 \vec{v}_{2}-3 \vec{v}_{1}\right)\right]+2\left(\dot{\vec{S}}_{1} \cdot \vec{S}_{2}\right)\left(\vec{S}_{2} \cdot \vec{n}\right)\left[\vec{S}_{1} \cdot\left(2 \vec{v}_{1}-3 \vec{v}_{2}\right)\right] \\
& -2\left[S_{1}^{2}-5\left(\vec{S}_{1} \cdot \vec{n}\right)^{2}\right]\left[\left(\vec{S}_{2} \cdot \vec{a}_{2}\right)\left(\vec{S}_{2} \cdot \vec{n}\right)+\left(\dot{\vec{S}}_{2} \cdot \vec{v}_{2}\right)\left(\vec{S}_{2} \cdot \vec{n}\right)+\left(\vec{S}_{2} \cdot \vec{v}_{2}\right)\left(\dot{\vec{S}}_{2} \cdot \vec{n}\right)\right] \\
& +2\left[S_{2}^{2}-5\left(\vec{S}_{2} \cdot \vec{n}\right)^{2}\right]\left[\left(\vec{S}_{1} \cdot \vec{a}_{1}\right)\left(\vec{S}_{1} \cdot \vec{n}\right)+\left(\dot{\vec{S}}_{1} \cdot \vec{v}_{1}\right)\left(\vec{S}_{1} \cdot \vec{n}\right)+\left(\vec{S}_{1} \cdot \vec{v}_{1}\right)\left(\dot{\vec{S}}_{1} \cdot \vec{n}\right)\right] \\
& -5\left[S_{2}^{2}-3\left(\vec{S}_{2} \cdot \vec{n}\right)^{2}\right]\left[\left(\dot{\vec{S}}_{1} \cdot \vec{v}_{2}\right)\left(\vec{S}_{1} \cdot \vec{n}\right)+\left(\vec{S}_{1} \cdot \vec{v}_{2}\right)\left(\dot{\vec{S}}_{1} \cdot \vec{n}\right)\right] \\
& +5\left[S_{1}^{2}-3\left(\vec{S}_{1} \cdot \vec{n}\right)^{2}\right]\left[\left(\dot{\vec{S}}_{2} \cdot \vec{v}_{1}\right)\left(\vec{S}_{2} \cdot \vec{n}\right)+\left(\vec{S}_{2} \cdot \vec{v}_{1}\right)\left(\dot{\vec{S}}_{2} \cdot \vec{n}\right)\right] \\
& +5\left(\vec{S}_{1} \cdot \vec{n}\right)\left(\dot{\vec{S}}_{1} \cdot \vec{n}\right)\left[S_{2}^{2}+7\left(\vec{S}_{2} \cdot \vec{n}\right)^{2}\right]\left(\vec{v}_{2} \cdot \vec{n}\right) \\
& -5\left(\vec{S}_{2} \cdot \vec{n}\right)\left(\dot{\vec{S}}_{2} \cdot \vec{n}\right)\left[S_{1}^{2}+7\left(\vec{S}_{1} \cdot \vec{n}\right)^{2}\right]\left(\vec{v}_{1} \cdot \vec{n}\right) \\
& +8\left(\vec{S}_{1} \cdot \vec{v}_{2}\right)\left(\vec{S}_{1} \cdot \vec{n}\right)\left(\dot{\vec{S}}_{2} \cdot \vec{S}_{2}\right)-8\left(\vec{S}_{2} \cdot \vec{v}_{1}\right)\left(\vec{S}_{2} \cdot \vec{n}\right)\left(\dot{\vec{S}}_{1} \cdot \vec{S}_{1}\right) \\
& -2\left(\vec{S}_{1} \cdot \vec{v}_{1}\right)\left(\vec{S}_{1} \cdot \vec{n}\right)\left[7\left(\dot{\vec{S}}_{2} \cdot \vec{S}_{2}\right)-5\left(\vec{S}_{2} \cdot \vec{n}\right)\left(\dot{\vec{S}}_{2} \cdot \vec{n}\right)\right] \\
& +2\left(\vec{S}_{2} \cdot \vec{v}_{2}\right)\left(\vec{S}_{2} \cdot \vec{n}\right)\left[7\left(\dot{\vec{S}}_{1} \cdot \vec{S}_{1}\right)-5\left(\vec{S}_{1} \cdot \vec{n}\right)\left(\dot{\vec{S}}_{1} \cdot \vec{n}\right)\right] \\
& +5\left(\dot{\vec{S}}_{1} \cdot \vec{S}_{1}\right)\left[S_{2}^{2}-7\left(\vec{S}_{2} \cdot \vec{n}\right)^{2}\right]\left(\vec{v}_{2} \cdot \vec{n}\right)-4\left(\dot{\vec{S}}_{1} \cdot \vec{S}_{1}\right)\left[S_{2}^{2}-5\left(\vec{S}_{2} \cdot \vec{n}\right)^{2}\right]\left(\vec{v}_{1} \cdot \vec{n}\right) \\
& -5\left[S_{1}^{2}-7\left(\vec{S}_{1} \cdot \vec{n}\right)^{2}\right]\left(\dot{\vec{S}}_{2} \cdot \vec{S}_{2}\right)\left(\vec{v}_{1} \cdot \vec{n}\right)+4\left[S_{1}^{2}-5\left(\vec{S}_{1} \cdot \vec{n}\right)^{2}\right]\left(\dot{\vec{S}}_{2} \cdot \vec{S}_{2}\right)\left(\vec{v}_{2} \cdot \vec{n}\right), \\
& L_{(3)}=+3\left(\dot{\vec{S}}_{1} \cdot \dot{\vec{S}}_{2}\right)\left(\vec{S}_{1} \cdot \vec{n}\right)\left(\vec{S}_{2} \cdot \vec{n}\right)-3\left(\dot{\vec{S}}_{1} \cdot \dot{\vec{S}}_{2}\right)\left(\vec{S}_{1} \cdot \vec{S}_{2}\right)+3\left(\dot{\vec{S}}_{1} \cdot \vec{n}\right)\left(\dot{\vec{S}}_{2} \cdot \vec{n}\right)\left(\vec{S}_{1} \cdot \vec{S}_{2}\right) \\
& +13\left(\dot{\vec{S}}_{1} \cdot \vec{S}_{1}\right)\left(\dot{\vec{S}}_{2} \cdot \vec{S}_{2}\right)-3\left(\dot{\vec{S}}_{1} \cdot \vec{S}_{2}\right)\left(\vec{S}_{1} \cdot \dot{\vec{S}}_{2}\right)+15\left(\dot{\vec{S}}_{1} \cdot \vec{n}\right)\left(\dot{\vec{S}}_{2} \cdot \vec{n}\right)\left(\vec{S}_{1} \cdot \vec{n}\right)\left(\vec{S}_{2} \cdot \vec{n}\right) \\
& -21\left(\dot{\vec{S}}_{1} \cdot \vec{n}\right)\left(\vec{S}_{1} \cdot \vec{n}\right)\left(\dot{\vec{S}}_{2} \cdot \vec{S}_{2}\right)-21\left(\dot{\vec{S}}_{1} \cdot \vec{S}_{1}\right)\left(\dot{\vec{S}}_{2} \cdot \vec{n}\right)\left(\vec{S}_{2} \cdot \vec{n}\right) \\
& +3\left(\vec{S}_{1} \cdot \dot{\vec{S}}_{2}\right)\left(\dot{\vec{S}}_{1} \cdot \vec{n}\right)\left(\vec{S}_{2} \cdot \vec{n}\right)+3\left(\dot{\vec{S}}_{1} \cdot \vec{S}_{2}\right)\left(\vec{S}_{1} \cdot \vec{n}\right)\left(\dot{\vec{S}}_{2} \cdot \vec{n}\right) \\
& +2\left[\dot{S}_{1}^{2}+\left(\ddot{\vec{S}}_{1} \cdot \vec{S}_{1}\right)\right]\left[S_{2}^{2}-3\left(\vec{S}_{2} \cdot \vec{n}\right)^{2}\right]+2\left[S_{1}^{2}-3\left(\vec{S}_{1} \cdot \vec{n}\right)^{2}\right]\left[\dot{S}_{2}^{2}+\left(\ddot{\vec{S}}_{2} \cdot \vec{S}_{2}\right)\right], \\
& L_{(4)}=+S_{1}^{2} S_{2}^{2}-4 S_{1}^{2}\left(\vec{S}_{2} \cdot \vec{n}\right)-4 S_{2}^{2}\left(\vec{S}_{1} \cdot \vec{n}\right)^{2}+\left(\vec{S}_{1} \cdot \vec{S}_{2}\right)^{2} \\
& -12\left(\vec{S}_{1} \cdot \vec{S}_{2}\right)\left(\vec{S}_{1} \cdot \vec{n}\right)\left(\vec{S}_{2} \cdot \vec{n}\right)+24\left(\vec{S}_{1} \cdot \vec{n}\right)^{2}\left(\vec{S}_{2} \cdot \vec{n}\right)^{2}, \\
& L_{(5)}=+30\left(\vec{S}_{1} \cdot \vec{n}\right)\left(\vec{S}_{1} \cdot \vec{S}_{2}\right)\left(\vec{S}_{2} \cdot \vec{n}\right)-57\left(\vec{S}_{1} \cdot \vec{n}\right)^{2}\left(\vec{S}_{2} \cdot \vec{n}\right)^{2} \\
& -3\left(\vec{S}_{1} \cdot \vec{S}_{2}\right)^{2}+9 S_{1}^{2}\left(\vec{S}_{2} \cdot \vec{n}\right)^{2}+S_{1}^{2} S_{2}^{2},
\end{aligned}
$$

and

$$
\begin{aligned}
L_{\mathrm{S}_{1}^{3} S_{2}}^{\mathrm{NLO}}= & C_{1\left(\mathrm{BS}^{3}\right)} \frac{G}{m_{1}^{2}}\left(\frac{L_{[1]}}{4 r^{5}}+\frac{L_{[2]}}{4 r^{4}}+\frac{L_{[3]}}{12 r^{3}}\right)+C_{1\left(\mathrm{BS}^{3}\right)} \frac{G^{2}}{m_{1} r^{6}} L_{[4]}+C_{1\left(\mathrm{BS}^{3}\right)} \frac{m_{2} G^{2}}{m_{1}^{2} r^{6}} L_{[5]} \\
& +C_{1\left(\mathrm{ES}^{2}\right)} \frac{G^{2}}{m_{1} r^{6}} L_{[6]}+\frac{3}{2} C_{1\left(\mathrm{ES}^{2}\right)} \frac{G}{m_{1}^{2} r^{4}} L_{[7]}+3 C_{1\left(\mathrm{ES}^{2}\right)} \frac{m_{2} G^{2}}{m_{1}^{2} r^{6}} L_{[8]},
\end{aligned}
$$

with the pieces:

$$
\begin{aligned}
L_{[1]}= & -2\left(\vec{S}_{1} \cdot \vec{S}_{2}\right)\left(\vec{S}_{1} \cdot \vec{v}_{1}\right)^{2}+2\left(\vec{S}_{1} \cdot \vec{S}_{2}\right)\left(\vec{S}_{1} \cdot \vec{v}_{1}\right)\left(\vec{S}_{1} \cdot \vec{v}_{2}\right) \\
& -30\left(\vec{S}_{1} \cdot \vec{S}_{2}\right)\left(\vec{S}_{1} \cdot \vec{v}_{1}\right)\left(\vec{S}_{1} \cdot \vec{n}\right)\left[\left(\vec{v}_{1}-\vec{v}_{2}\right) \cdot \vec{n}\right] \\
& +10\left(\vec{S}_{1} \cdot \vec{S}_{2}\right)\left(\vec{S}_{1} \cdot \vec{v}_{2}\right)\left(\vec{S}_{1} \cdot \vec{n}\right)\left[\left(\vec{v}_{1}-4 \vec{v}_{2}\right) \cdot \vec{n}\right] \\
& +\left(\vec{S}_{1} \cdot \vec{S}_{2}\right)\left[13\left(v_{1}^{2}+v_{2}^{2}\right)-29\left(\vec{v}_{1} \cdot \vec{v}_{2}\right)-20\left(\vec{v}_{1} \cdot \vec{n}\right)^{2}\right. \\
& \left.-20\left(\vec{v}_{2} \cdot \vec{n}\right)^{2}+25\left(\vec{v}_{1} \cdot \vec{n}\right)\left(\vec{v}_{2} \cdot \vec{n}\right)\right]
\end{aligned}
$$




$$
\begin{aligned}
& -5\left(\vec{S}_{1} \cdot \vec{n}\right)^{2}\left[13\left(v_{1}^{2}+v_{2}^{2}\right)-29\left(\vec{v}_{1} \cdot \vec{v}_{2}\right)-28\left(\vec{v}_{1} \cdot \vec{n}\right)^{2}\right. \\
& \left.-28\left(\vec{v}_{2} \cdot \vec{n}\right)^{2}+35\left(\vec{v}_{1} \cdot \vec{n}\right)\left(\vec{v}_{2} \cdot \vec{n}\right)\right] \\
& -15\left[3 S_{1}^{2}-7\left(\vec{S}_{1} \cdot \vec{n}\right)^{2}\right]\left(\vec{S}_{1} \cdot \vec{n}\right)\left(\vec{S}_{2} \cdot \vec{v}_{2}\right)\left[\left(\vec{v}_{1}-\vec{v}_{2}\right) \cdot \vec{n}\right] \\
& +5\left[3 S_{1}^{2}-7\left(\vec{S}_{1} \cdot \vec{n}\right)^{2}\right]\left(\vec{S}_{1} \cdot \vec{n}\right)\left(\vec{S}_{2} \cdot \vec{v}_{1}\right)\left[\left(4 \vec{v}_{1}-5 \vec{v}_{2}\right) \cdot \vec{n}\right] \\
& -15 S_{1}^{2}\left(\vec{S}_{1} \cdot \vec{n}\right)\left(\vec{S}_{2} \cdot \vec{n}\right)\left[3\left(v_{1}^{2}+v_{2}^{2}\right)-7\left(\vec{v}_{1} \cdot \vec{v}_{2}\right)-7\left(\vec{v}_{1} \cdot \vec{n}\right)\left(\vec{v}_{2} \cdot \vec{n}\right)\right] \\
& +35\left(\vec{S}_{1} \cdot \vec{n}\right)^{3}\left(\vec{S}_{2} \cdot \vec{n}\right)\left[3\left(v_{1}^{2}+v_{2}^{2}\right)-7\left(\vec{v}_{1} \cdot \vec{v}_{2}\right)-9\left(\vec{v}_{1} \cdot \vec{n}\right)\left(\vec{v}_{2} \cdot \vec{n}\right)\right] \\
& +50\left(\vec{S}_{1} \cdot \vec{v}_{1}\right)^{2}\left(\vec{S}_{1} \cdot \vec{n}\right)\left(\vec{S}_{2} \cdot \vec{n}\right)+40\left(\vec{S}_{1} \cdot \vec{v}_{2}\right)^{2}\left(\vec{S}_{1} \cdot \vec{n}\right)\left(\vec{S}_{2} \cdot \vec{n}\right) \\
& -90\left(\vec{S}_{1} \cdot \vec{v}_{1}\right)\left(\vec{S}_{1} \cdot \vec{v}_{2}\right)\left(\vec{S}_{1} \cdot \vec{n}\right)\left(\vec{S}_{2} \cdot \vec{n}\right) \\
& +25\left[S_{1}^{2}-7\left(\vec{S}_{1} \cdot \vec{n}\right)^{2}\right]\left(\vec{S}_{1} \cdot \vec{v}_{1}\right)\left(\vec{S}_{2} \cdot \vec{n}\right)\left[\left(\vec{v}_{1}-\vec{v}_{2}\right) \cdot \vec{n}\right] \\
& -\left[S_{1}^{2}-5\left(\vec{S}_{1} \cdot \vec{n}\right)^{2}\right]\left(\vec{S}_{1} \cdot \vec{v}_{1}\right)\left[\vec{S}_{2} \cdot\left(17 \vec{v}_{1}-11 \vec{v}_{2}\right)\right] \\
& +\left[S_{1}^{2}-5\left(\vec{S}_{1} \cdot \vec{n}\right)^{2}\right]\left(\vec{S}_{1} \cdot \vec{v}_{2}\right)\left[\vec{S}_{2} \cdot\left(19 \vec{v}_{1}-13 \vec{v}_{2}\right)\right] \\
& -5\left[S_{1}^{2}-7\left(\vec{S}_{1} \cdot \vec{n}\right)^{2}\right]\left(\vec{S}_{1} \cdot \vec{v}_{2}\right)\left(\vec{S}_{2} \cdot \vec{n}\right)\left[\left(7 \vec{v}_{1}-4 \vec{v}_{2}\right) \cdot \vec{n}\right], \\
& L_{[2]}=+2\left(\vec{S}_{1} \cdot \vec{S}_{2}\right)\left[S_{1}^{2}-10\left(\vec{S}_{1} \cdot \vec{n}\right)^{2}\right]\left(\vec{a}_{1} \cdot \vec{n}\right)-4\left(\vec{S}_{1} \cdot \vec{S}_{2}\right)\left[S_{1}^{2}-5\left(\vec{S}_{1} \cdot \vec{n}\right)^{2}\right]\left(\vec{a}_{2} \cdot \vec{n}\right) \\
& -3\left(\vec{S}_{1} \cdot \vec{v}_{1}\right)\left[S_{1}^{2}-5\left(\vec{S}_{1} \cdot \vec{n}\right)^{2}\right]\left(\dot{\vec{S}}_{2} \cdot \vec{n}\right)+4\left(\vec{S}_{1} \cdot \vec{v}_{2}\right)\left[S_{1}^{2}-5\left(\vec{S}_{1} \cdot \vec{n}\right)^{2}\right]\left(\dot{\vec{S}}_{2} \cdot \vec{n}\right) \\
& +\left(\dot{\vec{S}}_{1} \cdot \vec{v}_{2}\right)\left[S_{1}^{2}+5\left(\vec{S}_{1} \cdot \vec{n}\right)^{2}\right]\left(\vec{S}_{2} \cdot \vec{n}\right)+4\left(\vec{S}_{1} \cdot \vec{a}_{2}\right)\left[S_{1}^{2}-5\left(\vec{S}_{1} \cdot \vec{n}\right)^{2}\right]\left(\vec{S}_{2} \cdot \vec{n}\right) \\
& +\left[11 S_{1}^{2}-45\left(\vec{S}_{1} \cdot \vec{n}\right)^{2}\right]\left(\dot{\vec{S}}_{1} \cdot \vec{n}\right)\left(\vec{S}_{2} \cdot \vec{v}_{2}\right)+2\left(\dot{\vec{S}}_{1} \cdot \vec{S}_{2}\right)\left[S_{1}^{2}-10\left(\vec{S}_{1} \cdot \vec{n}\right)^{2}\right]\left(\vec{v}_{1} \cdot \vec{n}\right) \\
& -2\left[7 S_{1}^{2}-10\left(\vec{S}_{1} \cdot \vec{n}\right)^{2}\right]\left(\vec{S}_{1} \cdot \vec{n}\right)\left(\vec{S}_{2} \cdot \vec{a}_{1}\right)+5\left[3 S_{1}^{2}-7\left(\vec{S}_{1} \cdot \vec{n}\right)^{2}\right]\left(\vec{S}_{1} \cdot \vec{n}\right)\left(\dot{\vec{S}}_{2} \cdot \vec{n}\right)\left(\vec{v}_{1} \cdot \vec{n}\right) \\
& -\left(\vec{S}_{1} \cdot \dot{\vec{S}}_{2}\right)\left[S_{1}^{2}-5\left(\vec{S}_{1} \cdot \vec{n}\right)^{2}\right]\left(\vec{v}_{1} \cdot \vec{n}\right)+\left(\dot{\vec{S}}_{1} \cdot \vec{S}_{2}\right)\left[S_{1}^{2}+5\left(\vec{S}_{1} \cdot \vec{n}\right)^{2}\right]\left(\vec{v}_{2} \cdot \vec{n}\right) \\
& -2\left(\dot{\vec{S}}_{1} \cdot \vec{n}\right)\left[7 S_{1}^{2}-30\left(\vec{S}_{1} \cdot \vec{n}\right)^{2}\right]\left(\vec{S}_{2} \cdot \vec{v}_{1}\right)-4\left(\vec{S}_{1} \cdot \dot{\vec{S}}_{2}\right)\left[S_{1}^{2}-5\left(\vec{S}_{1} \cdot \vec{n}\right)^{2}\right]\left(\vec{v}_{2} \cdot \vec{n}\right) \\
& -5\left(\dot{\vec{S}}_{1} \cdot \vec{n}\right)\left[5 S_{1}^{2}-21\left(\vec{S}_{1} \cdot \vec{n}\right)^{2}\right]\left(\vec{S}_{2} \cdot \vec{n}\right)\left(\vec{v}_{2} \cdot \vec{n}\right)-\left[3 S_{1}^{2}-5\left(\vec{S}_{1} \cdot \vec{n}\right)^{2}\right]\left(\vec{S}_{1} \cdot \vec{n}\right)\left(\dot{\vec{S}}_{2} \cdot \vec{v}_{1}\right) \\
& +8\left(\vec{S}_{1} \cdot \vec{S}_{2}\right)\left[\left(\vec{S}_{1} \cdot \vec{a}_{1}\right)+\left(\dot{\vec{S}}_{1} \cdot \vec{v}_{1}\right)-5\left(\dot{\vec{S}}_{1} \cdot \vec{n}\right)\left(\vec{v}_{1} \cdot \vec{n}\right)\right]\left(\vec{S}_{1} \cdot \vec{n}\right) \\
& -10\left(\vec{S}_{1} \cdot \vec{S}_{2}\right)\left[\left(\dot{\vec{S}}_{1} \cdot \vec{v}_{2}\right)-\left(\dot{\vec{S}}_{1} \cdot \vec{n}\right)\left(\vec{v}_{2} \cdot \vec{n}\right)\right]\left(\vec{S}_{1} \cdot \vec{n}\right) \\
& +2\left(\vec{S}_{1} \cdot \vec{S}_{2}\right)\left(\dot{\vec{S}}_{1} \cdot \vec{n}\right)\left[\vec{S}_{1} \cdot\left(4 \vec{v}_{1}-5 \vec{v}_{2}\right)\right]+2\left(\vec{S}_{1} \cdot \vec{S}_{2}\right)\left(\dot{\vec{S}}_{1} \cdot \vec{S}_{1}\right)\left[\left(2 \vec{v}_{1}+\vec{v}_{2}\right) \cdot \vec{n}\right] \\
& -2\left[\left(\vec{S}_{1} \cdot \dot{\vec{S}}_{2}\right)-4\left(\dot{\vec{S}}_{1} \cdot \vec{S}_{2}\right)\right]\left(\vec{S}_{1} \cdot \vec{v}_{1}\right)\left(\vec{S}_{1} \cdot \vec{n}\right) \\
& -10\left[\left(\dot{\vec{S}}_{1} \cdot \vec{S}_{2}\right)-\left(\dot{\vec{S}}_{1} \cdot \vec{n}\right)\left(\vec{S}_{2} \cdot \vec{n}\right)\right]\left(\vec{S}_{1} \cdot \vec{v}_{2}\right)\left(\vec{S}_{1} \cdot \vec{n}\right) \\
& +2\left(\dot{\vec{S}}_{1} \cdot \vec{S}_{1}\right)\left(\vec{S}_{1} \cdot \vec{n}\right)\left[11\left(\vec{S}_{2} \cdot \vec{v}_{2}\right)+10\left(\vec{S}_{2} \cdot \vec{n}\right)\left(\vec{v}_{1} \cdot \vec{n}\right)-14\left(\vec{S}_{2} \cdot \vec{v}_{1}\right)-25\left(\vec{S}_{2} \cdot \vec{n}\right)\left(\vec{v}_{2} \cdot \vec{n}\right)\right] \\
& -2\left(\dot{\vec{S}}_{1} \cdot \vec{S}_{1}\right)\left[\vec{S}_{1} \cdot\left(2 \vec{v}_{1}-\vec{v}_{2}\right)\right]\left(\vec{S}_{2} \cdot \vec{n}\right)-2 S_{1}^{2}\left[\left(\vec{S}_{1} \cdot \vec{a}_{1}\right)+\left(\dot{\vec{S}}_{1} \cdot \vec{v}_{1}\right)\right]\left(\vec{S}_{2} \cdot \vec{n}\right) \\
& +10 S_{1}^{2}\left[\left(\vec{S}_{1} \cdot \vec{n}\right)\left(\vec{a}_{1} \cdot \vec{n}\right)+\left(\dot{\vec{S}}_{1} \cdot \vec{n}\right)\left(\vec{v}_{1} \cdot \vec{n}\right)\right]\left(\vec{S}_{2} \cdot \vec{n}\right), \\
& L_{[3]}=+\left(\dot{\vec{S}}_{1} \cdot \dot{\vec{S}}_{2}\right)\left[S_{1}^{2}-3\left(\vec{S}_{1} \cdot \vec{n}\right)^{2}\right]-9\left(\dot{\vec{S}}_{1} \cdot \vec{n}\right)\left[S_{1}^{2}-5\left(\vec{S}_{1} \cdot \vec{n}\right)^{2}\right]\left(\dot{\vec{S}}_{2} \cdot \vec{n}\right) \\
& -6\left(\dot{\vec{S}}_{1} \cdot \vec{n}\right)\left(\vec{S}_{1} \cdot \dot{\vec{S}}_{2}\right)\left(\vec{S}_{1} \cdot \vec{n}\right)-18\left(\dot{\vec{S}}_{1} \cdot \vec{S}_{1}\right)\left(\dot{\vec{S}}_{2} \cdot \vec{n}\right)\left(\vec{S}_{1} \cdot \vec{n}\right)+2\left(\dot{\vec{S}}_{1} \cdot \vec{S}_{1}\right)\left(\vec{S}_{1} \cdot \dot{\vec{S}}_{2}\right) \text {, }
\end{aligned}
$$




$$
\begin{aligned}
L_{[4]}= & -3\left(\vec{S}_{1} \cdot \vec{S}_{2}\right)\left[S_{1}^{2}-5\left(\vec{S}_{1} \cdot \vec{n}\right)^{2}\right]+2\left[9 S_{1}^{2}-20\left(\vec{S}_{1} \cdot \vec{n}\right)^{2}\right]\left(\vec{S}_{1} \cdot \vec{n}\right)\left(\vec{S}_{2} \cdot \vec{n}\right), \\
L_{[5]}= & -9\left(\vec{S}_{1} \cdot \vec{S}_{2}\right)\left[S_{1}^{2}-5\left(\vec{S}_{1} \cdot \vec{n}\right)^{2}\right]+51\left(\vec{S}_{1} \cdot \vec{n}\right)\left(\vec{S}_{2} \cdot \vec{n}\right) S_{1}^{2}-115\left(\vec{S}_{1} \cdot \vec{n}\right)^{3}\left(\vec{S}_{2} \cdot \vec{n}\right), \\
L_{[6]}= & -\left(\vec{S}_{1} \cdot \vec{S}_{2}\right)\left[S_{1}^{2}-9\left(\vec{S}_{1} \cdot \vec{n}\right)^{2}\right]+6\left(\vec{S}_{1} \cdot \vec{n}\right)\left[S_{1}^{2}-4\left(\vec{S}_{1} \cdot \vec{n}\right)^{2}\right]\left(\vec{S}_{2} \cdot \vec{n}\right), \\
L_{[7]}= & {\left[2 S_{1}^{2}\left(\vec{S}_{2} \cdot \vec{a}_{1}\right)+\left(\dot{\vec{S}}_{1} \cdot \vec{S}_{1}\right)\left(\vec{S}_{2} \cdot \vec{v}_{1}\right)-\left(\dot{\vec{S}}_{1} \cdot \vec{S}_{2}\right)\left(\vec{S}_{1} \cdot \vec{v}_{1}\right)\right]\left(\vec{S}_{1} \cdot \vec{n}\right) } \\
& +\left[2 S_{1}^{2}\left(\vec{a}_{1} \cdot \vec{n}\right)+\left(\dot{\vec{S}}_{1} \cdot \vec{S}_{1}\right)\left(\vec{v}_{1} \cdot \vec{n}\right)-\left(\dot{\vec{S}}_{1} \cdot \vec{n}\right)\left(\vec{S}_{1} \cdot \vec{v}_{1}\right)\right]\left[\vec{S}_{1} \cdot \vec{S}_{2}-5\left(\vec{S}_{1} \cdot \vec{n}\right)\left(\vec{S}_{2} \cdot \vec{n}\right)\right] \\
& -2\left(\vec{S}_{1} \cdot \vec{n}\right)\left(\vec{S}_{1} \cdot \vec{a}_{1}\right)\left(2\left(\vec{S}_{1} \cdot \vec{S}_{2}\right)-5\left(\vec{S}_{1} \cdot \vec{n}\right)\left(\vec{S}_{2} \cdot \vec{n}\right)\right), \\
L_{[8]}= & S_{1}^{2}\left[\vec{S}_{1} \cdot \vec{S}_{2}-4\left(\vec{S}_{1} \cdot \vec{n}\right)\left(\vec{S}_{2} \cdot \vec{n}\right)\right]-\left(\vec{S}_{1} \cdot \vec{n}\right)^{2}\left[2\left(\vec{S}_{1} \cdot \vec{S}_{2}\right)-5\left(\vec{S}_{1} \cdot \vec{n}\right)\left(\vec{S}_{2} \cdot \vec{n}\right)\right],
\end{aligned}
$$

as well as

$$
\begin{aligned}
L_{\mathrm{S}_{1}^{4}}^{\mathrm{NLO}}= & C_{1\left(\mathrm{ES}^{4}\right)} \frac{G m_{2}}{m_{1}^{3}}\left(\frac{L_{\{1\}}}{16 r^{5}}+\frac{L_{\{2\}}}{8 r^{4}}+\frac{L_{\{3\}}}{12 r^{3}}\right)-\frac{1}{8} C_{1\left(\mathrm{ES}^{4}\right)} \frac{G^{2} m_{2}}{r^{6} m_{1}^{2}} L_{\{4\}}-\frac{3}{8} C_{1\left(\mathrm{ES}^{4}\right)} \frac{G^{2} m_{2}^{2}}{r^{6} m_{1}^{3}} L_{\{5\}} \\
& +C_{1\left(\mathrm{BS}^{3}\right)} \frac{G^{2} m_{2}}{r^{6} m_{1}^{2}} L_{\{6\}}-\frac{1}{8} C_{1\left(\mathrm{ES}^{2}\right)}^{2} \frac{G^{2} m_{2}}{r^{6} m_{1}^{2}} L_{\{7\}} \\
& +\frac{1}{2} C_{1\left(\mathrm{BS}^{3}\right)} \frac{G m_{2}}{r^{4} m_{1}^{3}} L_{\{8\}}+C_{1\left(\mathrm{BS}^{3}\right)} \frac{G^{2} m_{2}^{2}}{r^{6} m_{1}^{3}} L_{\{9\}}+\frac{C_{1\left(\mathrm{E}^{2} \mathrm{~S}^{4}\right)}}{24} \frac{G^{2} m_{2}^{2}}{r^{6} m_{1}^{3}} L_{\{10\}},
\end{aligned}
$$

with the pieces:

$$
\begin{aligned}
L_{\{1\}}= & +12\left(\vec{S}_{1} \cdot \vec{v}_{1}\right)\left(\vec{S}_{1} \cdot \vec{v}_{2}\right)\left[S_{1}^{2}-5\left(\vec{S}_{1} \cdot \vec{n}\right)^{2}\right]-12\left(\vec{S}_{1} \cdot \vec{v}_{1}\right)^{2}\left[S_{1}^{2}-5\left(\vec{S}_{1} \cdot \vec{n}\right)^{2}\right] \\
& +20\left(\vec{S}_{1} \cdot \vec{n}\right)\left[3 S_{1}^{2}-7\left(\vec{S}_{1} \cdot \vec{n}\right)^{2}\right]\left[\left(\vec{S}_{1} \cdot \vec{v}_{1}\right)\left(\vec{v}_{1}-\vec{v}_{2}\right) \cdot \vec{n}-\left(\vec{S}_{1} \cdot \vec{v}_{2}\right)\left(\vec{v}_{1} \cdot \vec{n}\right)\right] \\
& +\left[3 S_{1}^{4}-30 S_{1}^{2}\left(\vec{S}_{1} \cdot \vec{n}\right)^{2}+35\left(\vec{S}_{1} \cdot \vec{n}\right)^{4}\right]\left[3 v_{1}^{2}+3 v_{2}^{2}-7\left(\vec{v}_{1} \cdot \vec{v}_{2}\right)\right] \\
& -15\left[S_{1}^{4}-14 S_{1}^{2}\left(\vec{S}_{1} \cdot \vec{n}\right)^{2}+21\left(\vec{S}_{1} \cdot \vec{n}\right)^{4}\right]\left(\vec{v}_{1} \cdot \vec{n}\right)\left(\vec{v}_{2} \cdot \vec{n}\right), \\
L_{\{2\}}= & -6\left(\dot{\vec{S}}_{1} \cdot \vec{n}\right)\left(\vec{S}_{1} \cdot \vec{v}_{2}\right)\left[S_{1}^{2}-5\left(\vec{S}_{1} \cdot \vec{n}\right)^{2}\right]-10\left(\dot{\vec{S}}_{1} \cdot \vec{n}\right)\left(\vec{S}_{1} \cdot \vec{n}\right)\left[3 S_{1}^{2}-7\left(\vec{S}_{1} \cdot \vec{n}\right)^{2}\right]\left(\vec{v}_{2} \cdot \vec{n}\right) \\
& -12\left(\dot{\vec{S}}_{1} \cdot \vec{S}_{1}\right)\left(\vec{S}_{1} \cdot \vec{n}\right)\left(\vec{S}_{1} \cdot \vec{v}_{2}\right)+6\left(\dot{\vec{S}}_{1} \cdot \vec{S}_{1}\right)\left[S_{1}^{2}-5\left(\vec{S}_{1} \cdot \vec{n}\right)^{2}\right]\left(\vec{v}_{2} \cdot \vec{n}\right) \\
& -2\left(\dot{\vec{S}}_{1} \cdot \vec{v}_{2}\right)\left(\vec{S}_{1} \cdot \vec{n}\right)\left[3 S_{1}^{2}-5\left(\vec{S}_{1} \cdot \vec{n}\right)^{2}\right]-S_{1}^{2}\left[S_{1}^{2}-5\left(\vec{S}_{1} \cdot \vec{n}\right)^{2}\right]\left(\vec{a}_{1} \cdot \vec{n}\right) \\
& -2\left(\vec{S}_{1} \cdot \vec{a}_{1}\right)\left(\vec{S}_{1} \cdot \vec{n}\right) S_{1}^{2}, \\
L_{\{3\}}= & 3\left(\ddot{\vec{S}}_{1} \cdot \vec{n}\right)\left(\vec{S}_{1} \cdot \vec{n}\right) S_{1}^{2}-\left[\dot{S}_{1}^{2}+\left(\overrightarrow{\vec{S}}_{1} \cdot \vec{S}_{1}\right)\right]\left[2 S_{1}^{2}-3\left(\vec{S}_{1} \cdot \vec{n}\right)^{2}\right] \\
& +12\left(\dot{\vec{S}}_{1} \cdot \vec{n}\right)\left(\dot{\vec{S}}_{1} \cdot \vec{S}_{1}\right)\left(\vec{S}_{1} \cdot \vec{n}\right)+3\left(\overrightarrow{\vec{S}}_{1} \cdot \vec{n}\right)^{2} S_{1}^{2}-4\left(\dot{\vec{S}}_{1} \cdot \vec{S}_{1}\right)^{2}, \\
L_{\{4\}}= & 3 S_{1}^{4}-30 S_{1}^{2}\left(\vec{S}_{1} \cdot \vec{n}\right)^{2}+35\left(\vec{S}_{1} \cdot \vec{n}\right)^{4}, \\
L_{\{5\}}= & 95\left(\vec{S}_{1} \cdot \vec{n}\right)^{4}-81 S_{1}^{2}\left(\vec{S}_{1} \cdot \vec{n}\right)^{2}+8 S_{1}^{4}, \\
L_{\{6\}}= & \left(\vec{S}_{1} \cdot \vec{n}\right)^{2}\left[3 S_{1}^{2}-5\left(\vec{S}_{1} \cdot \vec{n}\right)^{2}\right], \\
L_{\{7\}}= & {\left[S_{1}^{2}-3\left(\vec{S}_{1} \cdot \vec{n}\right)^{2}\right]^{2}, } \\
L_{\{8\}}= & {\left[2 S_{1}^{2}\left(\vec{a}_{1} \cdot \vec{n}\right)-2\left(\vec{S}_{1} \cdot \vec{n}\right)\left(\vec{S}_{1} \cdot \vec{a}_{1}\right)+\left(\dot{\vec{S}}_{1} \cdot \vec{S}_{1}\right)\left(\vec{v}_{1} \cdot \vec{n}\right)-\left(\dot{\vec{S}}_{1} \cdot \vec{n}\right)\left(\vec{S}_{1} \cdot \vec{v}_{1}\right)\right] } \\
& \times\left[S_{1}^{2}-5\left(\vec{S}_{1} \cdot \vec{n}\right)^{2}\right],
\end{aligned}
$$




$$
\begin{aligned}
L_{\{9\}} & =S_{1}^{4}-6 S_{1}^{2}\left(\vec{S}_{1} \cdot \vec{n}\right)^{2}+5\left(\vec{S}_{1} \cdot \vec{n}\right)^{4}, \\
L_{\{10\}} & =S_{1}^{4}-6 S_{1}^{2}\left(\vec{S}_{1} \cdot \vec{n}\right)^{2}+9\left(\vec{S}_{1} \cdot \vec{n}\right)^{4} .
\end{aligned}
$$

The result has been ordered according to the Wilson coefficients, mass ratios, and the total number/order of higher-order time derivatives. The higher-order time derivatives of both the velocity and the spin are to be removed following the procedure that was shown in [49] via variable redefinitions. Then the lengthy result will reduce to an ordinary action, and will significantly shrink. But before we handle the higher-order time derivatives in this sector, we should take into account additional contributions to this sector from lowerorder redefinitions of variables made at lower-order sectors, similar to what was detailed in section 6 of [14]. In a forthcoming publication we will provide the reduced effective action along with other important quantities and observables from this sector.

\section{Conclusions}

In this work we derived for the first time the complete NLO gravitational quartic-in-spin interaction of generic compact binaries. The derivation built on the EFT of gravitating spinning objects introduced in [14], and mainly on its recent extensions carried out in [4, 10], in which a new type of worldline couplings should be considered, and the effective action of a spinning particle should be extended to quadratic order in the curvature. This sector enters at the 5PN order for maximally-spinning compact objects, and together with the NNNLO quadratic-in-spin sector studied in [10], provides all conservative finite-size spin effects up to this high PN order.

Following the cubic-in-spin sector studied in [4], a careful intricate analysis was required to recover a new type of composite worldline couplings that emerge due the fact that the linear momentum can no longer be considered independent of the spin at these nonlinear higher-spin sectors. These new worldline couplings that contribute here are of cubic and quartic order in the spin. It is interesting to consider whether these new couplings can be thought of as the classical manifestation of the total angular momenta of a composite particle.

The analysis in this work shows clearly that the spin-dependent correction to the linear momentum will have to be taken into account at quadratic order as of the NLO quinticin-spin level, which corresponds to the gravitational Compton scattering with a quantum spin $s=5 / 2$. This may render such a derivation impossibly complex, if not ill-defined. This fundamental connection between the classical and the quantum theories with higher spin will be clarified in a forthcoming publication.

At the loop computational scale, there was no new nor special difficulty in this sector, and in fact this demonstrated once again that even-in-spin sectors are much easier to handle compared to odd-in-spin ones, a trend that is clear from inspecting table 1. Yet, a new conceptual feature in this sector was a first relevant operator that is quadratic in the curvature, and entails a new Wilson coefficient.

In order to find out what is the effect of the contributions with composite worldline couplings from section 4 , and of the new operator quadratic in the curvature, we will derive 
in a forthcoming publication the reduced action, the equations of motion, the Hamiltonian, the consequent gauge-invariant observables, and further theoretical quantities, which will provide self-consistency checks for the validity of the results.

\section{Acknowledgments}

ML has received funding from the European Union's Horizon 2020 research and innovation programme under the Marie Skłodowska-Curie grant agreements No. 847523 'INTERACTIONS' and No. 764850 'SAGEX', and from the Carlsberg Foundation. FT is supported by the Knut and Alice Wallenberg Foundation under grant KAW 2013.0235, and the Ragnar Söderberg Foundation (Swedish Foundations' Starting Grant).

Open Access. This article is distributed under the terms of the Creative Commons Attribution License (CC-BY 4.0), which permits any use, distribution and reproduction in any medium, provided the original author(s) and source are credited.

\section{References}

[1] LIGO Scientific, ViRgo collaboration, Observation of gravitational waves from a binary black hole merger, Phys. Rev. Lett. 116 (2016) 061102 [arXiv:1602.03837] [InSPIRE].

[2] L. Blanchet, Gravitational radiation from post-Newtonian sources and inspiralling compact binaries, Living Rev. Rel. 17 (2014) 2 [arXiv:1310.1528] [INSPIRE].

[3] A. Buonanno and T. Damour, Effective one-body approach to general relativistic two-body dynamics, Phys. Rev. D 59 (1999) 084006 [gr-qc/9811091] [InSPIRE].

[4] M. Levi, S. Mougiakakos and M. Vieira, Gravitational cubic-in-spin interaction at the next-to-leading post-Newtonian order, arXiv:1912.06276 [INSPIRE].

[5] M. Levi, A.J. Mcleod and M. Von Hippel, $N^{3} L O$ gravitational spin-orbit coupling at order $G^{4}$, arXiv : 2003.02827 [INSPIRE].

[6] A. Antonelli, C. Kavanagh, M. Khalil, J. Steinhoff and J. Vines, Gravitational spin-orbit coupling through third-subleading post-Newtonian order: from first-order self-force to arbitrary mass ratios, Phys. Rev. Lett. 125 (2020) 011103 [arXiv:2003.11391] [INSPIRE].

[7] S. Foffa, P. Mastrolia, R. Sturani, C. Sturm and W.J. Torres Bobadilla, Static two-body potential at fifth post-Newtonian order, Phys. Rev. Lett. 122 (2019) 241605 [arXiv: 1902.10571] [INSPIRE].

[8] J. Blümlein, A. Maier and P. Marquard, Five-loop static contribution to the gravitational interaction potential of two point masses, Phys. Lett. B 800 (2020) 135100 [arXiv: 1902.11180] [INSPIRE].

[9] S. Foffa and R. Sturani, Hereditary terms at next-to-leading order in two-body gravitational dynamics, Phys. Rev. D 101 (2020) 064033 [arXiv:1907.02869] [InSPIRE].

[10] M. Levi, A.J. Mcleod and M. Von Hippel, NNNLO gravitational quadratic-in-spin interactions at the quartic order in $G$, arXiv:2003.07890 [INSPIRE].

[11] D. Bini, T. Damour and A. Geralico, Binary dynamics at the fifth and fifth-and-a-half post-Newtonian orders, Phys. Rev. D 102 (2020) 024062 [arXiv:2003.11891] [INSPIRE]. 
[12] D. Bini, T. Damour, A. Geralico, S. Laporta and P. Mastrolia, Gravitational dynamics at $O\left(G^{6}\right)$ : perturbative gravitational scattering meets experimental mathematics, arXiv:2008.09389 [INSPIRE].

[13] M. Levi and J. Steinhoff, Next-to-next-to-leading order gravitational spin-squared potential via the effective field theory for spinning objects in the post-Newtonian scheme, JCAP 01 (2016) 008 [arXiv: 1506.05794] [INSPIRE].

[14] M. Levi and J. Steinhoff, Spinning gravitating objects in the effective field theory in the post-Newtonian scheme, JHEP 09 (2015) 219 [arXiv:1501.04956] [INSPIRE].

[15] M. Levi and J. Steinhoff, EFTofPNG: a package for high precision computation with the Effective Field Theory of Post-Newtonian Gravity, Class. Quant. Grav. 34 (2017) 244001 [arXiv: 1705.06309] [INSPIRE].

[16] B.M. Barker and R.F. O'Connell, Gravitational two-body problem with arbitrary masses, spins, and quadrupole moments, Phys. Rev. D 12 (1975) 329 [InSPIRE].

[17] W.D. Goldberger and I.Z. Rothstein, An effective field theory of gravity for extended objects, Phys. Rev. D 73 (2006) 104029 [hep-th/0409156] [INSPIRE].

[18] R.A. Porto, Post-Newtonian corrections to the motion of spinning bodies in NRGR, Phys. Rev. D 73 (2006) 104031 [gr-qc/0511061] [INSPIRE].

[19] E. Poisson, Gravitational waves from inspiraling compact binaries: The Quadrupole moment term, Phys. Rev. D 57 (1998) 5287 [gr-qc/9709032] [INSPIRE].

[20] N. Arkani-Hamed, T.-C. Huang and Y.-t. Huang, Scattering amplitudes for all masses and spins, arXiv:1709.04891 [INSPIRE].

[21] A. Guevara, Holomorphic classical limit for spin effects in gravitational and electromagnetic scattering, JHEP 04 (2019) 033 [arXiv:1706. 02314] [INSPIRE].

[22] A. Guevara, A. Ochirov and J. Vines, Scattering of spinning black holes from exponentiated soft factors, JHEP 09 (2019) 056 [arXiv: 1812.06895] [INSPIRE].

[23] M.-Z. Chung, Y.-T. Huang, J.-W. Kim and S. Lee, The simplest massive S-matrix: from minimal coupling to Black Holes, JHEP 04 (2019) 156 [arXiv:1812.08752] [INSPIRE].

[24] M.-Z. Chung, Y.-T. Huang and J.-W. Kim, Classical potential for general spinning bodies, JHEP 09 (2020) 074 [arXiv: 1908.08463] [INSPIRE].

[25] R. Aoude, K. Haddad and A. Helset, On-shell heavy particle effective theories, JHEP 05 (2020) 051 [arXiv: 2001.09164] [INSPIRE].

[26] M.-Z. Chung, Y.-t. Huang, J.-W. Kim and S. Lee, Complete Hamiltonian for spinning binary systems at first post-Minkowskian order, JHEP 05 (2020) 105 [arXiv: 2003. 06600] [INSPIRE].

[27] Z. Bern, A. Luna, R. Roiban, C.-H. Shen and M. Zeng, Spinning black hole binary dynamics, scattering amplitudes and effective field theory, arXiv:2005.03071 [INSPIRE].

[28] M. Levi and J. Steinhoff, Leading order finite size effects with spins for inspiralling compact binaries, JHEP 06 (2015) 059 [arXiv:1410.2601] [INSPIRE].

[29] M. Levi and J. Steinhoff, Complete conservative dynamics for inspiralling compact binaries with spins at fourth post-Newtonian order, arXiv:1607.04252 [INSPIRE].

[30] S. Hergt and G. Schaefer, Higher-order-in-spin interaction Hamiltonians for binary black holes from source terms of Kerr geometry in approximate ADM coordinates, Phys. Rev. D 77 (2008) 104001 [arXiv:0712.1515] [INSPIRE]. 
[31] R.A. Porto and I.Z. Rothstein, Next to leading order Spin(1)Spin(1) effects in the motion of inspiralling compact binaries, Phys. Rev. D 78 (2008) 044013 [Erratum ibid. 81 (2010) 029905] [arXiv:0804.0260] [INSPIRE].

[32] J. Steinhoff, S. Hergt and G. Schaefer, Spin-squared Hamiltonian of next-to-leading order gravitational interaction, Phys. Rev. D 78 (2008) 101503 [arXiv:0809.2200] [INSPIRE].

[33] S. Hergt and G. Schaefer, Higher-order-in-spin interaction Hamiltonians for binary black holes from Poincaré invariance, Phys. Rev. D 78 (2008) 124004 [arXiv:0809.2208] [INSPIRE].

[34] S. Hergt, J. Steinhoff and G. Schaefer, Reduced Hamiltonian for next-to-leading order Spin-Squared Dynamics of General Compact Binaries, Class. Quant. Grav. 27 (2010) 135007 [arXiv: 1002.2093] [INSPIRE].

[35] V. Vaidya, Gravitational spin Hamiltonians from the S matrix, Phys. Rev. D 91 (2015) 024017 [arXiv: 1410 . 5348] [INSPIRE].

[36] S. Marsat, Cubic order spin effects in the dynamics and gravitational wave energy flux of compact object binaries, Class. Quant. Grav. 32 (2015) 085008 [arXiv:1411.4118] [INSPIRE].

[37] J. Vines and J. Steinhoff, Spin-multipole effects in binary black holes and the test-body limit, Phys. Rev. D 97 (2018) 064010 [arXiv:1606.08832] [InSPIRE].

[38] M. Levi and J. Steinhoff, Next-to-next-to-leading order gravitational spin-orbit coupling via the effective field theory for spinning objects in the post-Newtonian scheme, JCAP 01 (2016) 011 [arXiv: 1506.05056] [INSPIRE].

[39] B. Kol and M. Smolkin, Non-relativistic gravitation: from Newton to Einstein and back, Class. Quant. Grav. 25 (2008) 145011 [arXiv:0712.4116] [INSPIRE].

[40] B. Kol, M. Levi and M. Smolkin, Comparing space+time decompositions in the post-Newtonian limit, Class. Quant. Grav. 28 (2011) 145021 [arXiv:1011.6024] [InSPIRE].

[41] M. Levi, Effective field theories of post-Newtonian gravity: a comprehensive review, Rept. Prog. Phys. 83 (2020) 075901 [arXiv: 1807.01699] [INSPIRE].

[42] M. Levi, Binary dynamics from Spin1-Spin2 coupling at fourth post-Newtonian order, Phys. Rev. D 85 (2012) 064043 [arXiv:1107.4322] [INSPIRE].

[43] M. Levi, Next to leading order gravitational spin-orbit coupling in an effective field theory approach, Phys. Rev. D 82 (2010) 104004 [arXiv:1006.4139] [INSPIRE].

[44] D. Bini, T. Damour and G. Faye, Effective action approach to higher-order relativistic tidal interactions in binary systems and their effective one body description, Phys. Rev. D 85 (2012) 124034 [arXiv:1202.3565] [INSPIRE].

[45] M. Levi, Next to leading order gravitational Spin1-Spin2 coupling with Kaluza-Klein reduction, Phys. Rev. D 82 (2010) 064029 [arXiv:0802.1508] [INSPIRE].

[46] D. Binosi and L. Theussl, JaxoDraw: a graphical user interface for drawing Feynman diagrams, Comput. Phys. Commun. 161 (2004) 76 [hep-ph/0309015] [INSPIRE].

[47] D. Binosi, J. Collins, C. Kaufhold and L. Theussl, JaxoDraw: a graphical user interface for drawing Feynman diagrams. Version 2.0 release notes, Comput. Phys. Commun. 180 (2009) 1709 [arXiv: 0811.4113] [INSPIRE].

[48] J.A.M. Vermaseren, Axodraw, Comput. Phys. Commun. 83 (1994) 45 [InSPIRE]. 
[49] M. Levi and J. Steinhoff, Equivalence of ADM Hamiltonian and Effective Field Theory approaches at next-to-next-to-leading order spin1-spin2 coupling of binary inspirals, JCAP 12 (2014) 003 [arXiv: 1408.5762] [INSPIRE].

[50] W. Tulczyjew, Motion of multipole particles in general relativity theory, Acta Phys. Polon. 18 (1959) 393.

[51] W. Dixon, Dynamics of extended bodies in general relativity. I. Momentum and angular momentum, Proc. Roy. Soc. Lond. A 314 (1970) 499.

[52] R. Schattner, The uniqueness of the center-of-mass in general relativity, Gen. Rel. Grav. 10 (1979) 395 [INSPIRE].

[53] R. Schattner, The center-of-mass in general relativity, Gen. Rel. Grav. 10 (1979) 377 [INSPIRE]. 